\title{
Die Regulierungskrise des öffentlich-rechtlichen Rundfunks
}

\author{
Henk Erik Meier
}

Die Regulierung des öffentlich-rechtlichen Rundfunks befindet sich in einer Krise. Fragen nach den Ursachen, dem Verlauf und den Ergebnissen dieser Krise des Regulierungsregimes steben im Mittelpunkt dieses Beitrags. Während die Rundfunkanstalten diese Regierungskrise auf die standortpolitischen Interessen einzelner Länder und die neoliberale Ideologie der Europäischen Kommission zurückfübren, argumentiert der folgende Beitrag anhand von Überlegungen aus der politikwissenschaftlichen Forschung zur Dynamik von Regulierungsregimen (bistorischer Institutionalismus, PrincipalAgent-Ansatz) und interpretiert die Krise als Ergebnis einer Kumulation institutioneller Defizite des Regulierungsregimes. Diese Defizite hatten einen schleichenden politischen Legitimitätsverlust zur Folge, der auf Grund des Zusammentreffens verschiedener Faktoren zur Eskalation der Auseinandersetzungen fübrte. Eine grundlegende institutionelle Reform des öffentlich-rechtlichen Rundfunks ist jedoch wegen hoher Veränderungsbürden ausgeblieben. Allerdings laufen die gegenwärtigen Vorschläge zur Reform des Gebübrenfestsetzungsverfabrens auf eine Suspendierung des bislang dominierenden Regulierungsprinzips der Programmautonomie hinaus.

Schlagwörter: Regulierung, Medienrecht, Medienpolitik, öffentlich-rechtlicher Rundfunk, Gebühren, Gebührenfestsetzung, Programmauftrag, Programmautonomie, historischer Institutionalismus, Principal-Agent-Ansatz

\section{Einleitung*}

Die gegenwärtige Krise der Regulierung des öffentlich-rechtlichen Rundfunks wird markiert durch eine langwierige politische Auseinandersetzung über die seit dem 1. April 2005 geltende Gebührenerhöhung sowie durch ein europäisches Beihilfeverfahren, das sich gegen die Finanzierung der deutschen Rundfunkanstalten richtet. Neu an diesen Auseinandersetzungen ist nicht, dass es eine politische Debatte um die Gebührenerhöhung und Kritik der Europäischen Kommission am deutschen Regulierungsregime gibt. Von einer Krise muss vielmehr auf Grund der Zuspitzung der Auseinandersetzungen gesprochen werden. Die Länder haben sich nicht damit begnügt, weiterhin an die Rundfunkanstalten zu appellieren, ihren Auftrag zu präzisieren, sondern gemeinsam die Risiken eines neuen Verfassungsgerichtsverfahrens in Kauf genommen, um drängende Fragen der Regulierung des öffentlich-rechtlichen Rundfunks zu lösen, obwohl ihre rundfunkpolitische Gestaltungsfreiheit damit weiter eingeschränkt werden könnte. Darüber hinaus kann von einer institutionellen Krise auf Grund des grundsätzlichen Charakters und der potenziellen Tragweite der Auseinandersetzungen gesprochen werden. In der gegenwärtigen Auseinandersetzung ist ein Grundpfeiler der deutschen Rundfunkpolitik umstritten, nämlich die Interpretation der Reichweite der Programmautonomie des öffentlich-rechtlichen Rundfunks im digitalen Zeitalter. Aus Sicht der von dieser institutionellen Krise primär betroffenen Akteure, der öffentlich-

* Der Autor dankt Frau Prof. Dr. Adrienne Heritier sowie vier anonymen Gutachtern für wertvolle Hinweise, die deutlich zur Verbesserung des Manuskriptes beigetragen haben. 
rechtlichen Anstalten und ihrer privaten Konkurrenten, steht die Regulierung des dualen Systems im digitalen Zeitalter vor einem entscheidenden Wendepunkt (vgl. nur: DLR, 2006; ZDF, 2006; VPRT, 2005). Legt man die von Ansell und Vogel (2006) vorgeschlagene Definition einer institutionellen Krise zu Grunde, so muss von einer Regulierungskrise gesprochen werden, weil gegenwärtig alle fundamentalen Regulierungsfragen gestellt werden. Einerseits sind die Kriterien der Regulierung umstritten. Kontrovers ist, wie weit die Programmautonomie der Rundfunkanstalten reicht und inwieweit ihre Angebotspolitik zumindest in den neuen Medien wieder stärker politischen Kriterien unterworfen werden soll. Während die Kritiker der letzten Gebührenerhöhung Vorschläge präsentiert haben, die sehr weit gehend in die Programmgestaltung der Anstalten eingegriffen hätten ${ }^{1}$, ist hier entscheidend, dass unions- und sozialdemokratisch geführte Länder gleichermaßen übereingekommen sind, zumindest die letzte Gebührenentscheidung insoweit zu „repolitisieren“, als die Begründung der Länder für ihre Abweichung von der Gebührenempfehlung der Kommission zur Ermittlung des Finanzbedarfs der Rundfunkanstalten (KEF) genuin politische Kriterien nennt, die vom verfassungsrechtlich verankerten Grundsatz der Bedarfsgerechtigkeit der Gebührenfestsetzung abweichen. ${ }^{2}$ Darüber hinaus werden sowohl in der Gebührenauseinandersetzung als auch im Rahmen des europäischen Beihilfeverfahrens die ebenso fundamentalen Fragen nach den Regulierungsakteuren, der Verteilung von Regulierungskompetenzen zwischen politisch-administrativen Ebenen sowie der Institutionalisierung des Regulierungsregimes aufgeworfen. Umstritten ist, inwieweit die Anstalten ihren Auftrag allein determinieren können oder inwieweit andere Regulierungsakteure (Länder, Europäische Kommission) Einfluss auf die Auftragsinterpretation nehmen können und ob die Institutionalisierung des Regulierungsregimes etwa im Hinblick auf eine Stärkung der externen Leistungsevaluation verändert werden sollte.

Die Auseinandersetzungen um die Gebührenerhöhung wurden als standortpolitisch motivierter „Basar in Absurdistan“ charakterisiert, auf dem „um ein paar Cent weniger“ gefeilscht und geltendes Verfassungsrecht mit Füßen getreten worden sei (Anschlag, 2004). Das Beihilfeverfahren der Wettbewerbsdirektion der Europäischen Kommission (DG Comp) gegen die öffentlich-rechtlichen Rundfunkanstalten wird dagegen als zy-

$1 \mathrm{Zu}$ nennen wäre hier die geforderte Zusammenlegung der TV-Kanäle Arte und 3sat, das Ende der digitalen TV-Kanäle sowie die Reduzierung der ARD-Hörfunkprogramme (zu diesen Forderungen vgl. epd medien, 12, November 2003).

2 In der Gebührenauseinandersetzung beharrten die Gebührenkritiker unter den Ländern darauf, dass die Abweichung von der Gebührenentscheidung der KEF erkennbar als „politische Lösung" gerechtfertigt werden sollte (epd medien, 16. Juni 2004). Tatsächlich stellen die Länder in ihrer Begründung zur Abweichung von der KEF-Empfehlung fest, dass die Gebührenempfehlung in das Umfeld einer deutlich angespannten wirtschaftlichen Lage falle, die große Herausforderungen und finanzielle Einschränkungen für alle Teile der Bevölkerung mit sich bringe. Jenseits aller Bedarfskalkulationen sei daher die Angemessenheit der Belastung des Gebührenzahlers zu hinterfragen. Indem die Länder auf ungenutzte Einsparpotenziale und die (politisch erzwungenen) Selbstverpflichtungen verwiesen, um ihre Kürzung zu rechtfertigen, haben sie den genuin medien- und ordnungspolitischen Charakter ihrer Intervention deutlich gemacht: „Schließlich haben die Regierungschefs der Länder auch die aktuelle Gesamtentwicklung der Aufgaben im dualen Rundfunksystem und im Wettbewerb der Medien insgesamt berücksichtigt, da die Höhe der Rundfunkgebühr auch in diesem Zusammenhang nicht außer Betracht gelassen werden darf.“ (Begründung zur Änderung des Rundfunkfinanzierungsstaatsvertrags, zu Nummer 4). Die KEF hat ausdrücklich auf den nichtbedarfsgerechten Charakter dieser Kriterien hingewiesen und daher verfassungsrechtliche Bedenken geltend gemacht (vgl. epd medien, 25. und 29. September 2004). 
nischer neo-liberaler Anschlag auf die verfassungsrechtlichen Grundfesten der deutschen Rundfunkordnung gegeißelt (Eberle, 2004). Nun ist weder die Relevanz standortpolitischer Interessen für die Gebührenauseinandersetzung noch der marktliberale „bias“ der europäischen Wettbewerbspolitik zu leugnen. Allerdings lässt sich mit dieser verkürzten Deutung nicht erklären, wie es zu einer ernsten politischen Legitimationskrise bzw. Regulierungskrise ${ }^{3}$ des öffentlich-rechtlichen Rundfunks kommen konnte, in der dieser die Unterstützung bisheriger politischer Bündnispartner teilweise verlor. Die Fragen nach Ursachen, Verlauf und Ergebnissen dieser Krise des Regulierungsregimes stehen daher im Mittelpunkt dieses Beitrags. Die These ist, dass die Regulierungskrise des öffentlich-rechtlichen Rundfunks nicht lediglich als Ergebnis einer kurzsichtigen und populistischen Standort- oder Liberalisierungspolitik, sondern als Resultat einer Kumulation institutioneller Defizite eines komplexen Regulierungsregimes interpretiert werden muss. Wenn also in diesem Beitrag die Rede von Regulierungsdefiziten ist, wird stets auf politische Maßstäbe rekurriert. Dabei geht es nicht darum, die verfassungsrechtlich abgesicherte Doktrin der Programmautonomie als Regulierungsdefizit zu charakterisieren. Vielmehr steht der Befund im Mittelpunkt, dass die Doktrin der Programmautonomie in ihrer breiten Interpretation durch den öffentlich-rechtlichen Rundfunk die Rundfunkanstalten zu einer Angebotspolitik inspiriert hat, die von den Ländern als politisch problematisch wahrgenommen wird. Die Länder wollen nicht generell mehr Einfluss auf die Rundfunkanstalten, etwa im Sinne einer parteipolitischen Instrumentalisierung, nehmen, als ihnen das Verfassungsgericht zugestehen möchte, sondern die Länder haben in den jüngsten Auseinandersetzungen deutlich gemacht, dass das gegenwärtige Regulierungsregime aus ihrer Perspektive durch ein Stenerungs- und Kontrolldefizit im Hinblick auf die Angebotspolitik der Anstalten gekennzeichnet ist. Wie die jüngste Gebührendebatte zeigt, bestehen aus Sicht der Länder die politisch problematischen Effekte des gegenwärtigen Regulierungsregimes primär darin, eine schwer legitimierbare Expansion des öffentlich-rechtlichen Rundfunks und stetig steigende Rundfunkgebühren zu begünstigen. Daneben sind die kommerziellen Aktivitäten der Anstalten und die von ihnen vorgenommene Gewichtung zwischen Public Service-Werten und wettbewerbspolitischen Zielen umstritten. Dabei geht es den Ländern nicht um einen Generalangriff auf die Programmautonomie, wohl aber um eine Neugewichtung zwischen gesetzlicher Fremd- und autonomer Selbstbestimmung der Anstalten.

Insgesamt soll in diesem Artikel ein genuin empirisches Erkenntnisinteresse verfolgt werden, und nicht das normative Anliegen, ein alternatives Regulierungsregime zu entwerfen. Die zentrale These lautet, dass die institutionellen Defizite des existierenden Regulierungsregimes einen schleichenden politischen Legitimitätsverlust des Regulierungsregimes zur Folge hatten. Die aktuelle Krise ist Ergebnis einer institutionell angelegten Tendenz zur Verselbständigung, der gegenüber das existierende Regulierungsregime keine ausreichenden Korrekturmechanismen vorsah. Die politische Legitimationskrise eskalierte schließlich, als spezifische Rahmenbedingungen zusammentrafen, die sich zum Nachteil des öffentlich-rechtlichen Rundfunks auswirkten und seinen Kritikern ein Gelegenheitsfenster schufen, in dem sie ihre Interessen durchsetzen konnten.

Zunächst werden im Folgenden die konzeptionellen Grundlagen der Argumentation

3 Wenn im Folgenden von Legitimitätsverlust die Rede ist, wird ausschließlich auf das gesunkene medienpolitische Vertrauen und nicht auf die gesellschaftliche Akzeptanz der Rundfunkanstalten abgehoben. Indikatoren für gesellschaftliche Legitimität wären die Akzeptanz der Gebührenhöhe sowie das Vertrauen in die sowie die Nutzung der Angebote des öffentlich-rechtlichen Rundfunks. 
vorgestellt. ${ }^{4}$ Dann wird skizziert, wie spezifische Bedingungen der Institutionengenese Defizite des Regulierungsregimes begünstigt haben. Nach einer Analyse der institutionellen Defizite werden Entwicklung und Eskalation des Legitimitätsverlust der Anstalten rekonstruiert. Anschließend wird auf die Implikationen der derzeitigen Reformvorschläge für die Zukunft des öffentlich-rechtlichen Rundfunks eingegangen. Das Fazit versucht sich an einer konzeptionellen Reflexion der Fallstudie.

\section{Die konzeptionellen Module zur Erklärung der Krise der Regulierung des öffentlich-rechtlichen Rundfunks}

Um der Komplexität des Steuerungsarrangements für den öffentlich-rechtlichen Rundfunk gerecht zu werden, wird der Begriff des „Regulierungsregimes“ herangezogen; dieser wird „defined as the full set of actors, institutions, norms and rules that are of importance for the process and the outcome of public regulation in a given sector" (Eberlein \& Grande, 2005, S. 91). Dieses Begriffskonzept hat den Vorteil, dass es die Aufmerksamkeit auf die vielfältigen und fragmentierten Einflussbeziehungen im Bereich des öffentlich-rechtlichen Rundfunks lenkt, die widersprüchliche und konkurrierende Steuerungsimpulse zur Folge haben können. Der hier verwendete Regulierungsbegriff bezieht sich auf die Definition, Überwachung und Sanktionierung des Angebotsverhaltens des öffentlich-rechtlichen Rundfunks im dualen Rundfunkmarkt.

Im Folgenden geht es nicht um die empirische Bestätigung monokausaler bivariater Hypothesen, sondern um die historische „Erklärung“ eines komplexen und historisch kontingenten Phänomens, der Krise des Regulierungsregimes des öffentlich-rechtlichen Rundfunks. Für die methodologische Bearbeitung derartiger „einmaliger“ und komplexer Phänomene empfiehlt die Policy-Forschung die Verwendung komplementärer konzeptioneller „Module“, deren Kombination es erlaubt, die für eine plausible historische „Erklärung “5 relevanten Dimensionen des jeweiligen komplexen Phänomens „vollständig“ zu berücksichtigen (Scharpf, 2000; Mayntz, 2002). Dieser methodologischen Anregung folgend soll eine „modulare“ Rekonstruktion versucht werden, indem Theoreme aus drei institutionalistischen Theoriediskursen verwendet werden. Gemeinsame Klammer dieser Ansätze ist die Fokussierung auf die institutionellen Grundlagen politischer Steuerungsprozesse und Koordinationsleistungen. Dabei verhalten sich die drei Theorieansätze insoweit komplementär zueinander, als sie es erlauben, die Genese institutioneller Defizite, ihren spezifischen Charakter und ihre Eskalation in einer Legitimationskrise zu thematisieren. Im Folgenden wird noch genauer auf die Gründe für die Rezeption der jeweiligen Ansätze eingegangen.

Um der Frage nachzugehen, warum ein etabliertes Regulierungsregime in beträchtliche Spannungen zu aktuellen Problemlagen geraten kann, wird der Ansatz des historischen Institutionalismus herangezogen, der sich explizit Aspekten der Genese und Persistenz politischer Institutionen widmet (Thelen, 1999; Pierson \& Skocpol, 2002; Pierson, 2004). Für diese konzeptionelle Entscheidung spricht, dass sich der historische Institutionalismus einerseits deutlich von funktionalistischen Ansätzen abhebt, die den Prozess der Institutionengenese als evolutionäre Selektion effizienter Koordinationslösungen konzeptionalisieren. Der historische Institutionalismus hat wiederholt auf die eingeschränkte Anwendbarkeit derartiger funktionalistischer Ansätze auf politische

4 Dieser Beitrag beruht auf früheren Arbeiten des Autors (Meier, 2003a; 2003b; 2005).

5 Natürlich bleibt eine derartige Erklärung im strengen epistemologischen Sinne unbewiesen, weil das Falsifikationsprinzip faktisch ausgeschlossen wird (Rueschemeyer \& Stephens, 1997). 
Phänomene hingewiesen, die wesentlich komplexeren Effizienzkriterien und Selektionsmechanismen unterliegen als wirtschaftliche Phänomene (s. auch: North, 1989). Andererseits unterscheidet sich der historische Institutionalismus auch deutlich von Ansätzen, die die Entstehung dysfunktionaler Institutionen auf das Nutzenkalkül interessenmaximierender Akteure zurückführen, die u. a. dysfunktionale Institutionen schaffen können, weil sich innerhalb dieser ihre Interessen besser verwirklichen lassen (Miller, 2000). Derartige Ansätze scheinen dem Untersuchungsgegenstand unangemessen, weil die Entwicklung des Regulierungsregimes im öffentlich-rechtlichen Rundfunk entscheidend durch das Bundesverfassungsgericht (BVerfG) geprägt worden ist. Die Handlungslogik des BVerfG, das sich um eine jeweils adäquate Auslegung der verfassungsrechtlichen Rundfunkfreiheit bemühen musste, folgt eher einer regel- und ethikorientieren „logic of appropriateness“ als einer interessenmaximierenden „logic of expected consequences“ (dazu: March \& Olsen, 1989). Über die Frage der Handlungslogik hinaus kann der Historische Institutionalismus der zentralen Rolle des BVerfG auch deshalb besser Rechnung tragen, weil er die temporale Dimension sozialer Prozesse und die kognitiven Beschränkungen bei der Schaffung politischer Institutionen betont. So geht er davon aus, dass die Konsequenzen institutioneller Entscheidungen in „dichtinstitutionalisierten“ Gesellschaften nie vollständig antizipiert werden können. Unintendierte Rückkopplungen und Interaktionseffekte sind daher die Folge. Widersprüche zwischen Regulierungsregimen und aktuellen Funktionserwartungen werden nach Auffassung des historischen Institutionalismus dann wahrscheinlich, wenn

(a) institutionelle Arrangements das Produkt singulärer Konfliktkonstellationen sind (Thelen, 1999);

(b) der Prozess der Institutionengenese dadurch geprägt ist, dass der „institutionelle Architekt“ strukturellen Beschränkungen hinsichtlich seiner Strategiefähigkeit unterliegt (Pierson, 2004), und

(c) Regulierungsregime ungeachtet ihrer fehlenden Leistungsfähigkeit eine erhebliche Persistenz aufweisen, weil die Veränderungskosten für institutionelle Anpassungen zu hoch sind (North, 1990).

Um die spezifischen Defizite des Regulierungsregimes für den öffentlich-rechtlichen Rundfunk zu erfassen, wird der institutionenökonomische Principal-Agent-Ansatz herangezogen. Die konzeptionelle Entscheidung für diesen Ansatz verdankt sich der Überlegung, dass sowohl die Länder als auch die Europäische Kommission die Steuerungsprobleme im öffentlich-rechtlichen Rundfunk als „Verselbständigung“ der Anstalten wahrnehmen und der Principal-Agent-Ansatz es erlaubt, derartige Tendenzen konsistent auf institutionelle Voraussetzungen zurückzuführen. Die Verwendung des Principal-Agent-Ansatzes impliziert, dass die Beziehung zwischen den Ländern, als Anstaltsträgern und Gewährleistungshaftern, und den Anstalten als Delegationsbeziehung verstanden wird, in der den Anstalten weitgehend selbständig die Erfüllung des „Programmauftrages“ zukommt. Wie im Folgenden zu zeigen sein wird, handelt es sich auf Grund der erheblich „verdünnten“ Verfügungsrechte der Länder gegenüber den Anstalten dabei freilich um eine analytische Vereinfachung, an der jedoch auf Grund ihrer heuristischen Ergiebigkeit festgehalten wird. Die heuristische Funktion des PrincipalAgent-Ansatzes im Rahmen dieses Beitrags besteht darin, als theoretisches Kontrastkonzept einer idealen Delegationsbeziebung verständlich zu machen, warum die verfassungsrechtlich verankerte Unabhängigkeit des öffentlich-rechtlichen Rundfunks auf Grund problematischer Anreize und schwach ausgeprägter alternativer Steuerungsmechanismen Interpretationen des Programmauftrages durch die Anstalten begünstigt, die die politische Legitimität der Anstalten aus Sicht der Länder mindern. 
Der Principal-Agent-Ansatz geht davon aus, dass jede Aufgabendelegation auf Grund der Informationsasymmetrien zwischen Auftraggeber („Prinzipal“) und Auftragnehmer („Agent“) unweigerlich Steuerungsprobleme aufwirft, weil nicht davon ausgegangen werden kann, dass Prinzipal und Agent die gleichen Präferenzen verfolgen. Gleichzeitig bestehen zwischen beiden Akteuren erhebliche Informationsasymmetrien (Moe, 1984; Gilardi \& Braun, 2002). Diese Bedingungen kommen dem Agenten entgegen, der die unvermeidlichen Informationsasymmetrien und Handlungsspielräume im Eigeninteresse nutzen kann (Jensen, 1983; Fama \& Jensen, 1983; Pratt \& Zeckhauser, 1985; Eisenhardt, 1989). Für ein genaueres Verständnis der Defizite des Regulierungsregimes des öffentlich-rechtlichen Rundfunks ist hilfreich, dass der Principal-AgentAnsatz es erlaubt, Hypothesen zur Wahrscheinlichkeit von Delegationsproblemen („agency loss“) und der Verselbständigung des Agenten („agency drift“) zu formulieren. Dabei ist für eine Anwendung des Ansatzes auf politische Delegationsprobleme essenziell, dass Beschränkungen auf Seiten des Principals ausdrücklich reflektiert werden müssen (Gilardi \& Braun, 2002). Eine Verselbständigung des Agenten ist nach dem Principal-Agent-Ansatz insbesondere dann wahrscheinlich, wenn

(a) auf Seiten des Prinzipals strukturelle Beschränkungen, etwa in Form „verdünnter“ Eigentumsrechte oder widersprüchlicher Präferenzen vorliegen (Alchian \& Demsetz, 1973),

(b) institutionelle Mechanismen zur Überwachung des Agenten für den Prinzipal nur schwer zu implementieren sind (Eisenhardt, 1989; McCubbins \& Schwartz, 1984),

(c) der Auftrag an den Agenten komplex und mehrdimensional ausfällt bzw. der Leistungsauftrag auf Grund seines langfristigen Charakters notwendigerweise unvollständig ist und daher dynamisch angepasst werden muss (sog. „relationale Verträge“; Hart \& Holmstrom, 1987; Holmstrom \& Milgrom, 1991), und

(d) der Agent mit stark widersprüchlichen Anreizen konfrontiert wird (Kiewiet \& McCubbins, 1991).

Um die Eskalation des Legitimitätsverlusts des Regulierungsregimes des öffentlichrechtlichen Rundfunks zu erklären, soll schließlich der „,contested governance“-Ansatz herangezogen werden, der unlängst von Chris Ansell und David Vogel (2006) entwickelt worden ist. Die Wahl dieses Eskalationsansatzes ist insoweit konsistent mit den beiden anderen konzeptionellen Modulen, insbesondere dem Historischen Institutionalismus, als dieser Ansatz davon ausgeht, dass Regulierungsregime zwar Defizite aufwerfen können, die Veränderungskosten aber so hoch sein können, dass gewisse Eskalationsvoraussetzungen erfüllt sein müssen, damit eine Revision des Regulierungsregimes stattfindet. Der „contested governance“-Ansatz widmet sich explizit der Frage, unter welchen Bedingungen die geringe Performanz von Regulierungsregimen „politisiert“ wird, so dass Gelegenheitsfenster geschaffen werden, die dramatische Veränderungen in politischen Debatten und Koalitionen zur Folge haben können (generell: Kingdon, 1995). In einem solchen Stadium der Krise des Regulierungsregimes, des „contested governance“, werden die fundamentalen Fragen nach den Kriterien der Regulierung, den Regulierungsakteuren, der Verteilung von Regulierungskompetenzen zwischen politisch-administrativen Ebenen sowie der Institutionalisierung des Regulierungsregimes aufgeworfen. Derartige Krisen treten nach Ansell und Vogel (2006) dann auf, wenn

(a) Regulierungsdefizite öffentlich skandalisiert werden oder die Öffentlichkeit sich zyklisch, aber intensiv mit Regulierungsfragen auseinandersetzt,

(b) skandalisierte Regulierungsdefizite mit längerfristigen institutionellen Schwächen, Interessenkonflikten oder konkurrierenden Regulierungsansätzen korrespondieren, 
(c) das Regulierungsregime sich durch eine geringe Responsivität gegenüber neuen Problemlagen auszeichnet.

Damit von einer institutionellen Krise im Sinne des „contested governance“ gesprochen werden kann, muss ein „Schneeballeffekt“ eintreten, der eine umfassende Reform des Regulierungsregimes zur Folge hat.

\section{Die Genese des Regulierungsregimes als einseitige Stärkung eines Regulierungsprinzips}

Im Folgenden soll die These unterstützt werden, dass die Defizite des Regulierungsregimes für den öffentlich-rechtlichen Rundfunk nicht zuletzt auf die besondere Genese des Regulierungsregimes, d. h. auf die zentrale Rolle des Bundesverfassungsgerichts bei der Institutionengenese, zurückgehen. Es wird argumentiert, dass die starke Rolle des BVerfG, aus der Sicht der politischen Akteure, zu einer Überdehnung der Programmautonomie als dominierendem Regulierungsprinzip geführt hat. Während die aktive Rolle des BVerfG bei der Ausgestaltung der deutschen Rundfunkordnung schwerlich zu bestreiten ist, soll hier nicht suggeriert werden, dass das Gericht nach Gutdünken seine eigene Rundfunkordnung entworfen habe. Vielmehr ist in Rechnung zu stellen, dass das Gericht in seiner Funktion als Interpret der verfassungsrechtlich verankerten Rundfunkfreiheit zwangsläufig in die aktive Rolle eines „Ersatzgesetzgebers“ gedrängt wurde, weil der politische Wettbewerb prekäre Anreize für politische Akteure zu kurzsichtigen Interventionen in den Mediensektor setzt. So ist die Rundfunkjudikatur erkennbar von der Erkenntnis getragen, dass Politiker einer starken Versuchung unterliegen, die Strukturen der Massenkommunikationsmittel einseitig zu ihren Gunsten zu beeinflussen und auf diese Weise die „Infrastruktur“ des demokratischen Prozesses zu beschädigen (Hoffmann-Riem, 1994). Zentral sowohl für die besondere Stellung des öffentlich-rechtlichen Rundfunks im politischen System der Bundesrepublik als auch die aktive Rolle des BVerfG bei der Genese des Regulierungsregimes sind daher die demokratietheoretisch einleuchtenden Bemühungen des Gerichts, die Funktion der Rundfunkanstalten im Prozess der demokratischen Meinungs- und Willensbildung zu sichern. Das Gebührenurteil von 1994 stellt insoweit nur den vorläufigen Endpunkt einer Entwicklung dar, in der das Verfassungsgericht die politischen Verfügungsrechte über den öffentlich-rechtlichen Rundfunk beschnitten hat, um einseitige Manipulationen der kommunikativen Infrastruktur der Demokratie zu verhindern. Allerdings läuft die Judikatur des Verfassungsgerichts auf die Suspendierung mehrheitsdemokratischer Auseinandersetzungen über die Rolle des öffentlichen Rundfunksektors hinaus. Der historische Institutionalismus legt die Hypothese nahe, dass die starke Rolle des Verfassungsgerichts das Auftreten von Spannungen zwischen der Performanz des Regulierungsregimes und aktuellen politischen Funktionserwartungen begünstigt:

Institutionengenese als kontingentes Produkt singulärer Konfliktkonstellationen: Zunächst ist festzuhalten, dass verfassungsgerichtliche Entscheidungen naturgemäß Antworten auf konkrete, z. T. singuläre Konfliktkonstellationen darstellen. Dabei hat das BVerfG in Reaktion auf situative Stimuli weit reichende Strukturfestlegungen getroffen, deren langfristige Auswirkungen schwer abschätzbar waren. Allerdings ist für die richterliche Rechtsfortbildung der Rundfunkfreiheit in Rechnung zu stellen, dass das BVerfG vor allem auf politische Übergriffsversuche auf den öffentlich-rechtlichen Rundfunk reagieren musste. Auf Grund dieser Kontingenz der verfassungsgerichtlichen Kasuistik avancierte die „Programmautonomie“ des öffentlich-rechtlichen Rundfunks zum entscheidenden Regulierungsprinzip. Diese Entwicklung der Verfassungsjudikatur 
ist einerseits durchaus nachvollziehbar, andererseits wurde in Reaktion auf spezifische Konfliktkonstellationen ein Regulierungsprinzip bis an die Grenzen der politischen Akzeptabilität übersteigert. Das Verfassungsgericht hat den öffentlich-rechtlichen Rundfunk zwar nicht kreiert, aber im ersten Fernsehurteil von 1961 den Grundsatz der Staatsferne des Rundfunks bekräftigt und das maßgeblich von den Alliierten geschaffene komplexe Aufsichts- und Kontrollsystem im öffentlich-rechtlichen Rundfunk verfassungsrechtlich legitimiert (BVerfGE 12, 205). In diesem Regulierungsregime sind die Aufsichtskompetenzen der Länder stark beschränkt und wird die „Fachaufsicht“ über die öffentlich-rechtlichen Rundfunkanstalten von den anstaltsinternen Aufsichtsgremien, Verwaltungs- und Rundfunkrat, die mit Vertretern gesellschaftlich relevanter Gruppen besetzt sind, wahrgenommen.

In Reaktion auf die medienpolitischen Bestrebungen der CDU-geführten Bundesländer in den achtziger Jahren, private Anbieter zuzulassen und die Aufgaben des öffentlich-rechtlichen Rundfunks zu beschneiden, entschloss sich das BVerfG, die sog. "Grundversorgung", die zwar auch von privaten Anbietern erbracht werden könnte, aber vor allem vom öffentlich-rechtlichen Rundfunk beansprucht wird, der Verfügungsgewalt des Gesetzgebers ganz zu entziehen, um die Erbringung wichtiger Informations- und Kommunikationsleistungen dauerhaft zu gewährleisten (BVerfGE 73, 118; BVerfGE 74, 297). Auch wenn das BVerfG bestimmte Angebote nicht der Grundversorgung zurechnete, ${ }^{6}$ ist festzuhalten, dass der Grundversorgungsbegriff nur unzureichend definiert und dynamisch angelegt wurde (dazu nur: Bullinger, 1999; Holznagel \& Vesting, 1999). Nachdem einige Länder wiederholt die Entscheidung über die Rundfunkgebühren zu medienpolitischen Interventionen nutzten, baute das BVerfG 1994 die Sonderstellung des öffentlich-rechtlichen Rundfunks weiter aus und stärkte die Programmautonomie der Anstalten noch einmal erheblich. Das Gericht räumte dem öffentlich-rechtlichen Rundfunk das Privileg der Mitwirkung an der Definition seines Funktions- und Aufgabenbereichs ein (Jarass, 1997), indem es die Entscheidung über die angemessene Erfüllung des Programmauftrages weitgehend den Anstalten überantwortete. Deren Einschätzungsprärogative bezieht sich sowohl auf Inhalt und Form der Programme als auch auf Programmstrukturen und "Anzahl und Umfang der Programme“ (BVerfGE 90, 60 [92]).

Strukturelle Beschränkungen des, institutionellen Architekten “: Während das BVerfG faktisch als „institutioneller Architekt“ agierte, indem es die Programmautonomie der Anstalten kontinuierlich ausbaute, sind die Möglichkeiten des Gerichts, ein umfassendes institutionelles Design einer Kommunikationsordnung ex nibilo zu entwerfen und detailliert auszugestalten, begrenzt. Das BVerfG hat sich zumeist darauf beschränkt, bestehende Institutionen partiell zu modifizieren und umzuprogrammieren. So enthält die Rechtsprechung des BVerfG zum Regulierungsregime des öffentlich-rechtlichen Rundfunks eine Reihe von „Leerstellen“, vor allem zu den genauen Konturen des Programmauftrages und den „Grenzen des Wachstums“ des öffentlich-rechtlichen Rundfunks

6 Natürlich ist festzuhalten, dass die „Grundversorgungsrechtsprechung“ Sparten- und Zielgruppenkanäle als „,jenseits der Grundversorgung“ charakterisiert hat (BVerfGE 74, 297 [332, 345-6]). Andererseits wirft der dynamische Charakter der Grundversorgung große Interpretationsprobleme aus. So sprach das BVerfG bereits im zweiten Grundversorgungsurteil von der notwendigen Weiterentwicklung des öffentlich-rechtlichen Programmauftrages in Reaktion auf technische Entwicklung und neue Formen und Inhalte (BVerfGE 74, 297 [324-5]; 83, 238 [298]). Inzwischen ist der Begriff der Grundversorgung durch den weniger missverständlichen, weil erkennbarer zukunftsoffen angelegten Begriff des Funktionsauftrages ersetzt worden (siehe etwa: Hoffmann-Riem, 2000). 
(Neun, 2002). Hier ist vor allem relevant, dass die erhebliche Stärkung der Programmautonomie der Anstalten obne eine Stärkung der selbstregulativen Mechanismen in den Anstalten oder die Schaffung einer auftragsorientierten externen Fremdevaluation erfolgte. ${ }^{7}$ So ist in der Verfassungsrechtsprechung bereits angelegt, was Ladeur (2000) für prozedurale Konzeptionen des öffentlich-rechtlichen Funktionsauftrages konstatiert, nämlich eine gefährliche Nähe zur tautologischen Aussage, dass der Public Service das ist, was die Anstalten als solchen definieren.

Hobe Veränderungskosten des Regulierungsregimes: Die starke Rolle des BVerfG erhöht die Kosten für die Anpassung des Regulierungsregimes an veränderte Problemlagen erheblich. Da die Anstalten sich auf verfassungsrechtliche Garantien berufen können, kann jeder Versuch eines politischen Eingriffs in das Regulierungsregime zu neuen verfassungsgerichtlichen Auseinandersetzungen führen. Diese können für die Länder erhebliche politische Kosten - in Form weiterer Einschränkungen ihrer rundfunkpolitischen Gestaltungsmittel - mit sich bringen.

Resümierend kann an dieser Stelle festgehalten werden, dass verschiedene Charakteristika der Institutionengenese im Bereich des öffentlich-rechtlichen Rundfunks das Auftreten von Spannungen zwischen dem existierenden Regulierungsregime und aktuellen Funktionserfordernissen begünstigen. Diese betreffen insbesondere das Problem der verfassungsgerichtlich eingeforderten, dynamischen Konkretisierung des Aufgabenfelds des öffentlich-rechtlichen Rundfunks (dazu: Hoffmann-Riem, 2000). Während das BVerfG spezifischen strukturellen Beschränkungen unterliegt, erhöht seine zentrale Rolle die Kosten für die Anpassung des Regulierungsregimes. Ein erhöhter Anpassungsbedarf ergibt sich jedoch zwangsläufig aus der Teilderegulierung und der technologischen Dynamik des audio-visuellen Sektors. Zu den relevanten Fragen zählen u. a. die Rolle des öffentlich-rechtlichen Rundfunks in einem teilderegulierten Rundfunksystem, d. h. ob und auf welche Angebots- und Zuschauersegmente sich der öffentliche Rundfunk konzentrieren soll, ob er in einen Qualitäts- und/oder Zuschauermarktwettbewerb mit Privatanbietern treten soll, ob er mit Gebührengeldern auf den Beschaffungsmärkten mit den Privaten konkurrieren soll und ob die besonderen Qualitätsverpflichtungen der Anstalten kodifiziert und einer öffentlichen Kontrolle unterliegen sollen. Die technologische Konvergenz macht schließlich Entscheidungen darüber erforderlich, an welchen technologischen Entwicklungen die Rundfunkanstalten teilhaben sollten (vgl. etwa: Hargreaves Heap, 2005), wobei diese neuen Technologien das Potenzial haben, zu einer Fülle individualisierter und fragmentierter Leistungsangebote zu führen und die „Integration“ als Funktionsleistung des öffentlichen Rundfunks fragwürdig werden zu lassen (Ladeur, 2000).

\section{Die Tendenz zur „agency drift“ als Defizit des Regulierungsregimes}

Mit Hilfe des analytischen Instrumentariums des Principal-Agent-Ansatzes soll nun der Nachweis erbracht werden, dass die Erhebung der Programmautonomie zum dominierenden Regulierungsprinzip eine Tendenz zur politisch nicht akzeptierbaren Interpretation des Programmauftrags durch die Anstalten bzw. zur „agency drift“ begünstigt.

Strukturelle Beschränkungen auf Seiten des Prinzipals: Wie bereits angedeutet, fügen sich die Anstalten auf Grund der verfassungsgerichtlichen Stärkung der „Programmautonomie“ nur bedingt in die Systematik des Principal-Agent-Ansatzes. Korrekterweise

7 Die existierende externe Fremdevaluation bezog sich vorrangig auf das Finanzgebahren der Anstalten, das in eingeschränkter Weise von KEF und Rechnungshöfen bewertet wurde und wird. 
wären die öffentlich-rechtlichen Rundfunkanstalten nicht als „Agenten“, sondern als „Treuhänder" zu charakterisieren, denen gegenüber der öffentliche bzw. politische Prinzipal, d. h. die Länder, wesentliche politische Verfügungsrechte verloren hat und nur über eingeschränkte Kontrollrechte gebietet (Majone, 2001; Gilardi \& Braun, 2002). Im Ergebnis dieser Stärkung der Programmautonomie sehen sich die Länder mit einer umfassenden finanziellen Gewährleistungspflicht für die Rundfunkanstalten konfrontiert, ohne dass sie deren Aufgaben determinieren könnten (oder dass diese in einer operablen Form festgeschrieben wären). Jeder Versuch einer gesetzlichen Fixierung des Aufgabenbereiches der Rundfunkanstalten fällt nun dem Generalverdacht des verfassungswidrigen Eingriffs in die Programmautonomie anheim (Neun, 2002). Die Verfassungsrechtsprechung favorisiert vielmehr deutlich legislative Ausweitungen des Programmauftrages (Ory, 1994). ${ }^{8}$

Der Verlust politischer Verfügungsrechte der Länder wird insbesondere an dem vom BVerfG entworfenen Gebührenfestsetzungsverfahren deutlich, das die Initiative für Gebührenerhöhungen konsequent den Anstalten überantwortet. Deren Finanzbedarfsanmeldungen dürfen von der nun entpolitisierten KEF lediglich im Hinblick auf Wirtschaftlichkeit und Sparsamkeit geprüft werden (BVerfGE 90, 60 [102-3]). Entscheidungen zum Programmumfang oder ordnungspolitische Wertungen darf die KEF nicht vornehmen. Die Landtage sollen nach Vorstellung des BVerfG die Gebührenempfehlung der KEF schlicht ratifizieren und dürfen nur „aus Gesichtspunkten des Informationszugangs und der angemessenen Belastung der Rundfunkteilnehmer" von ihr abweichen (BVerfGE 90, 60 [102-3]), wobei die herrschende Lehre diese Ausführungen als Sozialverträglichkeitsklausel interpretiert (Hesse, 1999).

Über diese verfassungsrechtlichen Beschränkungen hinaus ist festzuhalten, dass die Handlungsfähigkeit der Bundesländer gegenüber den Rundfunkanstalten faktisch durch die Strukturen des rundfunkpolitischen Verhandlungssystems sowie die widersprüchlichen Präferenzen der Länder begrenzt wird. Deutsche Rundfunkpolitik muss in einem multilateralen Verhandlungssystem formuliert werden, in dem sich jedes Land in der Rolle eines Vetospielers befindet. Da im Hinblick auf die Regulierung des öffentlichrechtlichen Rundfunks lange Zeit eine relativ klare parteipolitische Konfliktlinie existierte zwischen CDU-geführten Ländern, die eher dem privaten Rundfunk zugeneigt sind, und sozialdemokratisch geführten Ländern, die den öffentlich-rechtlichen Rundfunk als Gegengewicht zu den Privatmedien ansehen (Gellner, 1990; Tonnemacher, 1996; Spalleck, 2001), zeichnete sich das Verhandlungssystem durch eine relativ hohe Status quo-Orientierung im Hinblick auf eine Reform des Regulierungsregimes aus. Allerdings ist festzuhalten, dass nach der Teilderegulierung parteipolitische Präferenzen teilweise durch standortpolitische Erwägungen überlagert wurden. So war für die Erosion klarer parteipolitischer Konfrontation entscheidend, dass sich Nordrhein-Westfalen als (damaliges) SPD-Land zunehmend mit den medienpolitischen Interessen des Bertelsmann-Konzerns bzw. der RTL Group identifiziert hat (dazu auch: Stock, 1997). In der jüngsten Gebührenauseinandersetzung hat diese standortpolitische Unterstützung des Privatfunks offenbar die traditionelle Loyalität der nordrhein-westfälischen

8 Nach der Verfassungsjudikatur dürfen legislative Eingriffe in die Rundfunkfreiheit nicht der Beschränkung, sondern allein der Sicherung der Rundfunkfreiheit dienen. Dies ist nach Auffassung des BVerfG nur dann gegeben, wenn ausgestaltende Regelungen die Rundfunkfreiheit verbessern oder zumindest gleichwertig sichern (BVerfGE 74, 297 [334]). Ob etwa Programmzahlbeschränkungen verfassungsgemäß sind, ist Gegenstand intensiver verfassungsrechtlicher Debatten (Hoffmann-Riem, 2000). 
SPD zum WDR weitgehend in den Hintergrund gedrängt. Schließlich ist für die komplexe Präferenzstruktur der Länder festzuhalten, dass diese die Rundfunkgebühr auch als Finanzierungsinstrument für verschiedenste politische Projekte genutzt haben. Dies hat es den Anstalten erlaubt, Angebotsausweitungen als Beitrag zur nationalen oder regionalen Kultur- und Bildungspolitik oder zu industriepolitischen Projekten wie der Digitalisierung zu legitimieren. ${ }^{9}$

Problematische Überwachung und Sanktionierung des Agenten: Die PrincipalAgent-Literatur unterscheidet zwischen zwei Möglichkeiten der Überwachung des Agenten, einmal ergebnisorientierten („performance oriented“) und zum anderen verhaltensorientierten („behaviour oriented“) Überwachungsregimen, wobei ergebnisorientierte Überwachungsregime als effizienter gelten (Eisenhardt, 1989). Diese zielen auf eine Messung der Leistung des Agenten anhand möglichst eindeutiger Performanzindikatoren ab. Eine solche objektivierte Leistungskontrolle des öffentlich-rechtlichen Rundfunks gestaltet sich auf Grund der Komplexität der Leistungsvorgaben und der Probleme bei der Messung von Programmqualität freilich außerordentlich schwierig (s. u.). Zudem kannte das Regulierungsregime des öffentlich-rechtlichen Rundfunks eine externe performanzorientierte Überwachung des Angebotsverhaltens der Rundfunkanstalten, etwa in Form einer performanz- bzw. „zielorientierten“ Rechnungslegung, lange Zeit nicht (König, 1983). In Reaktion auf das Gebührenurteil haben die Länder freilich versucht, ergebnis- und verhaltensorientierte Steuerungs- und Überwachungsmechanismen innerhalb der Anstalten zu stärken. Der Impuls für diese Anpassungen des Regulierungsregimes ging dabei von den Rundfunkanstalten aus, die das Legitimitationsproblem, das aus einer weitgehenden Selbstdefinition ihres Programmauftrags resultiert, registriert haben. Die Anstalten haben versucht, die Vorbehalte gegenüber ihrer drohenden Verselbständigung zu relativieren, indem sie das Modell einer „prozeduralen Steuerung der Rundfunkentwicklung“ dahingehend weiterentwickelt haben, dass sie die zentrale Steuerungsaufgabe der binnenpluralistischen Aufsichtsgremien nicht nur in Fragen der Programmqualität und der Ausgewogenheit der Berichterstattung, sondern auch der Anpassung des Programmauftrages an neue Wettbewerbsbedingungen und technologische Entwicklungen herausgearbeitet haben. ${ }^{10}$ Das ent-

9 Im Verlauf der Gebührenauseinandersetzung präsentierte die ARD eine Aufstellung derartiger von den Ländern legitimierter oder eingeforderter Aktivitäten. Die ARD erwähnte u. a. die Digitalisierung, die Film- und Kulturförderung, die Finanzierung von Orchestern, Chören und Big Bands (vgl. epd medien, 24. Januar 2004). Während die Länder ihre Landesrundfunkanstalten traditionell als Mäzene der regionalen Kultur- oder Bildungspolitik agieren lassen, wird die Nutzung der Rundfunkgebühr als industriepolitischer Finanzierungsquelle am gegenwärtig umstrittensten Technologieprojekt deutlich, das aus Gebührenmitteln finanziert wird: dem digitalen Hörfunk. Obwohl die Nachfrageentwicklung dramatisch hinter den Prognosen zurückgeblieben ist und selbst die ARD eine Aufgabe des Projekts erwogen hat, halten einige Länder daran fest (s. nur: epd medien, 25. Februar 2004).

10 So gab das ZDF bspw. das sog. „Holznagel-Gutachten“ (Holznagel, 1999) in Auftrag, um auf das medienpolitische Unbehagen an der Stärkung der Programmautonomie zu reagieren. Das ZDF-Management, insbesondere die Intendanz, plädierte für eine Weiterentwicklung der Richtlinienkompetenz des Fernsehrates dahingehend, dass dieser der Anstalt ein „Pflichtenheft" vorgeben sollte, zu dem das ZDF in regelmäßigen Abständen in synoptischer Weise Stellung nehmen sollte. Dabei begriff die Führungsspitze des ZDF das „Holznagel-Gutachten“ explizit als Instrument der „geistigen Aufrüstung“ in der medienpolitischen Auseinandersetzung. Die Implementation dieser Überlegungen verzögerte sich jedoch, weil das ZDF-Management fürchtete, dass überprüfbare Selbstbindungen für die Beschneidung von Entwicklungschancen 
scheidende, verfassungsrechtlich unbedenkliche Steuerungsinstrument ist in diesem Modell die „Selbstbindung“ der Anstalten über nachprüfbare „codes of conduct“ o. Ä., die von den Aufsichtsgremien sanktioniert werden (Jarass, 1997; Holznagel, 1999). Angesichts der Tatsache, dass einige Aufsichtsgremien bereits früher die Kompetenz besaßen, Programmrichtlinien zu erlassen, stellt dies nur eine zeitgemäße und systemlogische Interpretation des Binnenpluralismus dar, die von den Aufsichtsgremien allerdings ein qualitativ anderes Steuerungshandeln verlangt (dazu detailliert: Meier, 2003a). ${ }^{11}$ Der im Jahr 2004 neu gefasste Rundfunkstaatsvertrag nimmt auf dieses Modell insoweit explizit Bezug, als er die Anstalten auffordert, Satzungen und Richtlinien zur näheren Ausgestaltung ihres jeweiligen Auftrags zu erlassen und über die Auftragserfüllung in zweijährigen Abständen zu berichten (\$11 IV RfStV 2004). Während diese prozeduralen Innovationen durchaus das Potenzial haben könnten, Steuerungswirkungen über „Selbstbindungen“ zu entfalten, auch wenn $\mathbb{1 1}$ Absatz 4 keinerlei Sanktionen festschreibt (!), bleibt festzuhalten, dass die bisherigen Erfahrungen mit „Selbstbindungen“ eher Skepsis nähren. Die jetzige Fassung des Rundfunkstaatsvertrages ist ein Ergebnis der Unzufriedenheit der Länder mit früheren Reaktionen der Anstalten auf die Aufforderung, Selbstverpflichtungen zu präsentieren. ${ }^{12}$ Darüber hinaus sind die ersten Berichte zur Auftragserfüllung der Anstalten selbst von wohlwollenden Beobachtern auf Grund des Fehlens konkreter Festlegungen nahezu zynisch kommentiert worden (Anschlag, 2004; Lilienthal, 2004b). Schließlich ist mit Blick auf die Effektivität von Sanktionsmechanismen festzuhalten, dass die binnenpluralistischen Aufsichtsgremien stets über ein einschneidendes Sanktionspotenzial verfügten, dies aber nur bedingt nutzten, um so etwas wie überprüfbare Selbstbindungen von den Anstalten einzufordern (Meier, 2003a).

Wird von der abzuwartenden Effektivität der Selbstbindungen abgesehen, stehen den Ländern neben dem riskanten Versuch einer legislativen Auftragsbeschränkung nur „strukturelle Eingriffe“ in Struktur und Organisation des öffentlich-rechtlichen Rundfunks, z. B. die Kündigung des ARD- oder ZDF-Staatsvertrages, offen (Bethge, 1996). Dabei handelt es sich zwar um ein sehr scharfes Sanktionsinstrument, seine Inanspruchnahme wäre jedoch mit erheblichen politischen Kosten und möglicherweise weiteren verfassungsrechtlichen Risiken verbunden (Meier, 2005).

Komplexe Auftragsstruktur der Anstalten: Es ist weithin unbestritten, dass der „Programmauftrag" der Rundfunkanstalten außerordentlich komplex ist und erhebliche Zielambiguitäten enthält. Der so genannte einfachgesetzliche „Programmauftrag“ stellt einen spannungsreichen Mix einzelner Zielvorgaben dar und enthält bspw. Verpflichtungen zur Erbringung von Vermittlungsleistungen, die von den Anstalten als Verpflichtung zur Massenattraktivität ausgelegt werden ${ }^{13}$, zur Minderheitenberücksichti-

genutzt werden oder die Komplexität des Leistungsauftrags sich gegen die Anstalt wenden könnten (vgl. dazu mit Zitaten aus Interviews: Meier, 2003a, S. 493-4).

$11 \mathrm{Im}$ Hinblick auf frühere durch die Gremien erlassene Richtlinien wurde festgestellt, dass diese zwar den Programmauftrag ergänzen, aber kaum konkretisieren (vgl. Pantenburg, 1996, S. 103).

12 Nach der Drohung eines Gebührenvetos durch den sächsischen Landtag im Jahr 2000 wurden die Anstalten verpflichtet, künftig allen Landtagen über ihre Haushaltsführung und Entwicklungsperspektiven zu berichten und den Grundversorgungsauftrag „eindeutig“ zu definieren (epd medien, 20. Dezember 2000; $\ 5$ a RFinStV 2002).

13 Der Programmauftrag enthält keine direkten Verpflichtungen zur Massenattraktivität, aber bspw. fordert der ZDF-Staatsvertrag vom ZDF, bestimmte Vermittlungsleistungen zu erbringen $(\$ 5 \mathrm{ZDF}-\mathrm{StV})$. In Verbindung mit der verfassungsrechtlich verankerten Integrationsfunktion der Anstalten (BVerfGE 47, 198 [225]) werden diese Vermittlungsziele von Management 
gung, zu informierenden, bildenden und kulturellen Funktionen und Standards. Diese Zielvorgaben bleiben überwiegend abstrakt und deklaratorisch, so dass eine „Ergebnissteuerung" der Anstalten außerordentlich schwierig ist (Ossenbühl, 1981; Kayser, 1993). Die Verfassungsjudikatur hat diesen „Programmauftrag“ noch einmal überformt und ausgeweitet. Entscheidend ist, dass das Gericht eine Bestands- und Entwicklungsgarantie für den öffentlich-rechtlichen Rundfunk ausgesprochen hat (BVerfGE 83, 238 [298]), die sich an der publizistischen Konkurrenzfähigkeit gegenüber privaten Anbietern und der Belebung des inländischen Gesamtangebotes orientieren soll (BVerfGE 87, 181 [202-3]). Daher handelt es sich beim öffentlich-rechtlichen „Programmauftrag“ um einen „relationalen Auftrag“, der vom Agenten teilweise autonom angepasst werden kann. Die verfassungsgerichtlich eingeforderte „Zukunftsoffenheit“ des öffentlichrechtlichen Auftrages hat zur Folge, dass sich normative Maßstäbe für eine unzulässige Expansion der Rundfunkanstalten nur äußerst schwer formulieren lassen (instruktiv: Hoffmann-Riem, 2000). Ungeachtet ihrer gebührenträchtigen Wettbewerbsziele sind die Anstalten auch zu Wirtschaftlichkeit und Sparsamkeit verpflichtet, ohne dass freilich geklärt wurde, wie sich Wirtschaftlichkeitserwägungen von Programmgestaltungsfragen trennen lassen. Schlussendlich sieht die Finanzierungskonzeption der Anstalten eine Werbefinanzierung und kommerzielle Aktivitäten vor, die prekäre Effekte für das Programmangebot öffentlicher Fernsehanbieter haben können (Blumler, 1993). Im Hinblick auf ihre Auftragsstruktur sind die öffentlich-rechtlichen Rundfunkanstalten daher als „multitask agents“ zu verstehen, zwischen deren einzelnen Auftragsdimensionen Konflikte und komplexe Interdependenzen bestehen (Holmstrom \& Milgrom, 1991).

Widersprüchliche Anreize auf Seiten der Anstalten: Die Tendenz zur „agency drift“ wird im Bereich des öffentlich-rechtlichen Rundfunks durch eine prekäre Anreizstruktur des Treuhänders verschärft. Während die Verfassungsjudikatur das entscheidende Steuerungsproblem im öffentlich-rechtlichen Rundfunk in der Sicherung von Unabhängigkeit und Neutralität verortet und auf eine professionelle Selbstregulierung vertraut (Stock, 2001), muss festgehalten werden, dass im öffentlich-rechtlichen Rundfunk nicht nur Zielambiguitäten, sondern auch rivalisierende Anreize existieren. Das verfassungsgerichtliche Steuerungskonzept ignoriert, dass alle Organisationen organisationsindividuelle Bestandserhaltungsziele haben, die zu beträchtlichen Zielverschiebungen führen können (Warner \& Havens, 1968; Perrow, 1986). Ohne suggerieren zu wollen, dass sich die Anstalten allein an diesen Bestandserhaltungszielen orientieren, ist empirisch belegt, dass sie eine ausgeprägte Furcht vor einer Marginalisierung auf dem $\mathrm{Zu}$ schauermarkt haben, da diese die Politik zu „strukturellen Eingriffen“ ermuntern könnte (Brandt, 1989; Bartels, 1997; Meier, 2003a). Diese organisationsindividuellen Bestandserhaltungsziele haben zur Folge, dass die Anstalten versuchen, sich in allen Angebotssegmenten $\mathrm{zu}$ engagieren, und in ihren Gebührenanmeldungen kurzfristige Einnahmeziele dominieren (Meier, 2005).

Zusammenfassend kann an dieser Stelle festgehalten werden, dass erhebliche strukturelle Beschränkungen auf Seiten des Prinzipals, schwach ausgeprägte Überwachungsund Sanktionierungsmechanismen, eine komplexe Auftragsstruktur sowie widersprüchliche Anreize auf Seiten der Anstalten, insbesondere starke Bestandserhaltungsinteressen, eine „Verselbständigung“ des öffentlich-rechtlichen Rundfunks begünstigen. Auf das Problem der „agency drift“ finden sich in der Judikatur des BVerfG frei-

und Aufsichtsgremien indirekt als Aufforderung interpretiert, zuschauerattraktive Programme anzubieten, um derartige Vermittlungs- und Integrationsleistungen überhaupt erbringen zu können (vgl. Meier, 2003a, S. 460-1). 
lich nur wenig Antworten. Insgesamt ist das bestehende Regulierungsregime einseitig darauf ausgerichtet, die Existenz eines starken öffentlich-rechtlichen Rundfunksektors zu gewährleisten. Es begünstigt auf Grund der problematischen Anreizstruktur der Anstalten insbesondere eine Expansion des Programmangebotes und der kommerziellen Aktivitäten der Rundfunkanstalten.

\section{Schleichender politischer Legitimitätsverlust}

Grundthese dieses Beitrags ist, dass die gegenwärtige Krise des Regulierungsregimes Ergebnis eines schleichenden politischen Legitimationsverlustes ist, die aus dem Eindruck einer institutionell begünstigten, stetigen Verselbständigung der Anstalten resultiert. In diesem Abschnitt soll daher herausgearbeitet werden, dass die Funktionalität des Regulierungsregimes seit längerem angezweifelt worden ist.

So wurde vor allem in den sechziger und siebziger Jahren kritisiert, dass - im Gegensatz zum Vertrauen des BVerfG in das binnenpluralistische Regulierungsregime die zu erheblichen Anteilen mit Parteipolitikern besetzten Aufsichtsgremien offensichtlich eine repressive Wirkung auf die Rundfunkjournalisten ausübten und einen umfassenden Personal-, Themen- und Meinungsproporz erzwangen (Fritz, 1977; Ladeur, 1978; Hennig, 1981). Zwischen der Realität des Binnenpluralismus und seiner normativ-ideologischen Rechtfertigung klafften erhebliche Lücken (Prodoehl, 1989; s. a. Schulz, 2002). Allerdings hat die Dualisierung offenbar zu einem Wandel der Aufsichtspraxis und einem Autonomiegewinn für die gesamte Rundfunkpublizistik geführt (Jarren, 2001), der darauf zurückgeht, dass der öffentlich-rechtliche Rundfunk durch die Dualisierung einen entscheidenden Teil seiner Definitionsmacht verloren hat und die Parteipolitiker einsehen mussten, dass der Proporzjournalismus zunehmend zuschauermarktunverträglich wurde (Meier, 2003a).

Wesentlich schwerwiegender für die politische Legitimität des Regulierungsregimes ist jedoch, dass die Dualisierung des Rundfunkmarktes die Frage aufwarf, ob das binnenpluralistische Regulierungsregime den neuen Steuerungsherausforderungen des dualen Rundfunkmarktes gewachsen war, d. h. ob die Aufsichtsgremien eine wettbewerbsbedingte Nivellierung des öffentlich-rechtlichen Programmangebotes - also eine „agency drift" der Rundfunkanstalten - verhindern konnten. In der wissenschaftlichen Debatte überwog erkennbar die Skepsis. Eine Reihe von Studien unterstützt die Einschätzung, dass die Rundfunkgremien gegenüber dem Management der Anstalten, d. h. in diesem Kontext: gegenüber Intendanz, Direktorenebene und zentralen Strategie- und Planungseinheiten, nur über eine sehr eingeschränkte Strategiefähigkeit verfügen (Hoffmann, 1975; Kepplinger \& Hartmann, 1989; Dussel, 1995; Meier, 2003a). Die mit der Dualisierung aufkommende und heftig diskutierte Konvergenzthese ist insoweit Ausdruck eines fehlenden Vertrauens in das existierende Regulierungsregime, als sie der besonderen Organisationsform der Anstalten keine Relevanz für deren Angebotsverhalten zumaß. Die Konvergenzthese ging davon aus, dass öffentlich-rechtliche und private Programmangebote dank der Konkurrenz auf dem Zuschauermarkt in Richtung auf ein unterhaltungsorientiertes Mischangebot konvergieren würden (Schatz et al., 1989; Schatz, 1994). In der medienpolitischen Interpretation lief die Konvergenzthese auf den Vorwurf hinaus, dass die Anstalten klassische Public Service-Werte zu Gunsten von Marktanteilszielen aufgeben würden. Tatsächlich konnte empirisch nachgewiesen werden, dass sich bspw. im ZDF nach der Dualisierung ein neuer "kompetitiver Konsens“ zwischen Management und Aufsichtsgremien eingestellt hat. Weil die Rundfunkräte die Aufrechterhaltung des öffentlich-rechtlichen Rundfunks und seiner Akzeptanz zuneh- 
mend als Vorbedingung dafür ansahen, dass hochwertige Programme überhaupt noch angeboten und nachgefragt wurden, identifizierten sich die Räte in wachsendem Maße mit den Wettbewerbszielen und der Unternehmenspolitik der Anstalt. Zudem hatten die Räte dem Management auf Grund der zunehmenden Komplexität des Fernsehmarktes wenig eigene strategische Überlegungen entgegenzuhalten. Sie akzeptierten daher, dass Einschaltquotenkalküle einen zentralen Einfluss auf die Angebotspolitik des ZDF erlangten (Meier, 2003a). Die empirische Programmforschung hat belegt, dass die Rundfunkanstalten in erheblichem Maße Gestaltungs- und Präsentationsformen ihrer privaten Konkurrenten übernahmen (Pfetsch, 1996; Marcinkowski \& Bruns, 1996; Bruns \& Marcinkowski, 1996; Krüger, 1992, 1996). Ungeachtet dessen hat eine weitgehende Angleichung von Programmprofilen, etwa gemessen an Unterhaltungs- und Informationsanteilen, nicht stattgefunden, auch wenn anspruchsvolle und Minderheitenprogramme an die Ränder des öffentlich-rechtlichen Programms gedrängt wurden. Dafür scheint ausschlaggebend, dass für die Anstalten vom Zuschauer- und Beschaffungsmarkt durchaus Anreize ausgingen, ein informationsorientiertes Programmprofil zu verfolgen. Ebenso war dem Management bewusst, dass es auf Grund der politischen Abhängigkeit der Anstalten die Selbstdarstellungsinteressen der politischen Klasse zu berücksichtigen hatte (Meier, 2003b). Auf Grund der unbestreitbaren Veränderungen in der Programmpolitik der Anstalten ist es aber nicht verwunderlich und für die politische Legitimation der Anstalten außerordentlich folgenreich, dass der vergröberte Konvergenzvorwurf als negatives Deutungsschema die Debatten um die öffentlich-rechtliche Angebotspolitik (Krüger, 2001) und damit mittelbar auch um die Performanz des Regulierungsregimes nachhaltig prägt. So wurde auch im Kontext der Gebührenauseinandersetzung der Standardvorwurf erhoben, die Anstalten hätten ihr spezifisches Angebotsprofil aufgegeben (s. nur: Stoiber, 2004; Simonis, 2004).

\section{Expansionsdebatte und Eskalationsvoraussetzungen}

Während die Konvergenzdebatte geeignet war, das Vertrauen in die Performanz des Regulierungsregimes zu erschüttern, bildete eine sich intensivierende Expansionsdebatte den thematischen Hintergrund der gegenwärtigen Eskalation des politischen Legitimitätsverlusts des Regulierungsregimes. Im Folgenden soll gezeigt werden, dass die gegenwärtige Eskalation der Regulierungskrise auf die Erfüllung der von Ansell und Vogel (2006) identifizierten Eskalationsvoraussetzungen zurückgeht.

Zunächst soll jedoch verdeutlicht werden, dass die Expansionspolitik der Anstalten aus ihrem komplexen Zielsystem und ihrer widersprüchlichen Anreizstruktur resultiert. Bereits in einem frühen Entwicklungsstadium des dualen Systems zeichnete sich ab, dass Management und Aufsichtsgremien der Rundfunkanstalten daran gingen, die existierenden Zielkonflikte durch eine Expansion und Diversifizierung des Programmangebots zu „lösen“, um Minderheitenpräferenzen in speziellen Programmangeboten zu berücksichtigen (Meier, 2003a). Die Anstalten gingen zudem von einer unaufhaltsamen Segmentierung des Zuschauerverhaltens aus, die eine Angebotsdiversifikation zur Sicherung der Zuschauerakzeptanz des öffentlich-rechtlichen Rundfunks unabdingbar machen würde (ZDF, 1994; Stolte, 1997). Die Stärkung der Programmautonomie ermöglichte den Anstalten dabei eine beträchtliche Expansionspolitik, von 1992 bis 2002 konnten sie ihr Programmangebot verdreifachen (Abb. 1). Inzwischen verfügen die deutschen Rundfunkanstalten über ein jährliches Gebührenaufkommen von rund 6,7 Mrd. $€$ (KEF, 2004, Tz. 285) und ein Gesamtbudget von über 8 Mrd. € im Jahr 2004. Nach den bis 2002 verfügbaren Statistiken wies Deutschland kontinuierlich den größ- 
Abbildung 1: Entwicklung der Sendeleistung des öffentlich-rechtlichen Fernsehens in Millionen Sendeminuten

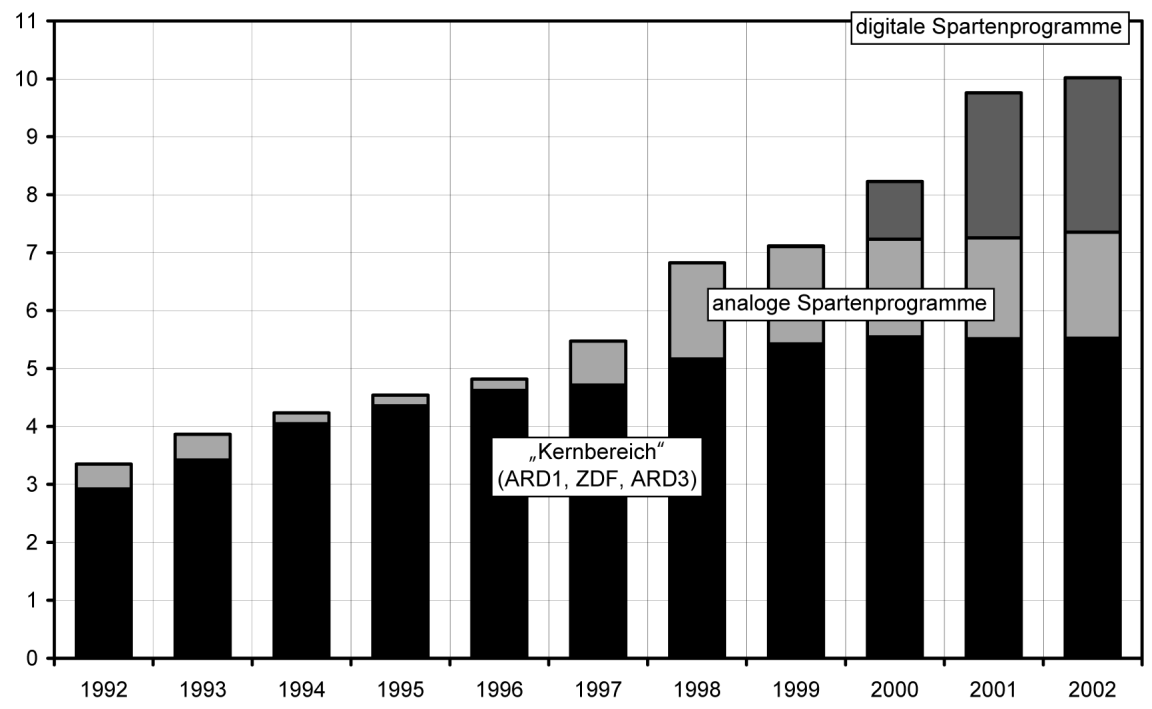

Quelle: 14. KEF-Bericht, Tz. 24; www.kef-online.de/inhalte/bericht14/14bericht.pdf

ten öffentlichen Rundfunksektor Europas sowie den höchsten Anteil öffentlicher Finanzierung im Fernsehen auf (vgl. Mattern \& Künstner, 1998; Deiss, 2002; Europäische Kommission, 2003).

Indikator dafür, dass zumindest einige der Prinzipale der Anstalten diese Entwicklung als „agency drift“ wahrnahmen, ist, dass die expansive Angebotspolitik kontinuierlich auf medienpolitischen Widerstand traf, insbesondere jener Länder, die aus standortpolitischen Gründen dem privaten Rundfunk nahe stehen. So wurden in den Gebührenrunden 1996 und 2000 sowohl die Effizienzbemühungen der Anstalten als auch die Legitimität ihrer Expansionspolitik bezweifelt. Innerhalb des multilateralen Verhandlungssystems der Länder konnten sich die Kritiker der Anstalten jedoch nicht durchsetzen. Einerseits hielt die Mehrzahl der sozialdemokratisch geführten Länder an ihrer grundsätzlichen Affinität zum öffentlich-rechtlichen Rundfunk fest und blockierte Einschnitte in das öffentlich-rechtliche Programmangebot. Andererseits „tauschten“ die Standortländer der Privatsender schließlich den Verzicht auf einschneidende strukturelle Eingriffe in den öffentlich-rechtlichen Rundfunk gegen Zugeständnisse bei der Regulierung des privaten Rundfunksektors (vgl. etwa: Stock, 1997).

Für die ernsthafte Krise des Regulierungsregimes im Jahr 2004 war entscheidend, dass die von Ansell und Vogel (2006) identifizierten Eskalationsbedingungen erfüllt waren:

(1) Zyklische Aufmerksamkeit und Skandalisierung von Regulierungsdefiziten: Die für das Jahr 2004 turnusmäßig anstehende Gebührenentscheidung verschaffte den Defiziten des Regulierungsregimes jene zyklische Aufmerksamkeit, die eine besondere Intensität der öffentlichen Debatte nach sich ziehen kann. Die zeitliche Kontextualisierung 
der unpopulären „Steuererhöhung“ war für die Anstalten diesmal besonders nachteilig. Das Begehren nach einer Gebührenerhöhung um 13 Prozent fiel mit kontrovers diskutierten Einschnitten in die sozialen Sicherungssysteme zusammen. Dies warf die Frage auf, warum die öffentlich-rechtlichen Rundfunkanstalten von den allgemeinen Sparzwängen ausgeschlossen sein sollten (Frankfurter Allgemeine Sonntagszeitung, 4. Januar 2004). Für die Skandalisierung von Regulierungsdefiziten sorgte schließlich die Aufdeckung rechtswidriger Schleichwerbepraktiken im öffentlich-rechtlichen Rundfunk. Der publizistische Anspruch der Anstalten und das Vertrauen in die binnenpluralistische Steuerung, insbesondere der kommerziellen Aktivitäten der Anstalten, wurden spürbar beschädigt (Kammann, 2003; Lilienthal, 2004a, 2005). Die Gebührenkritiker konnten vorbringen, dass die eigentlichen Gefahren dem öffentlich-rechtlichen Rundfunk von internen Fehlentwicklungen drohten (Stoiber, 2004, S. 4).

(2) Längerfristige Regulierungsdefizite, Konfliktlinien und konkurrierende Regulierungsansätze: Die skandalisierten Regulierungsdefizite korrespondierten sowohl mit längerfristigen Interessenkonflikten als auch mit einer Rivalität unterschiedlicher Regulierungsansätze innerhalb des europäischen Mehrebenensystems.

Zunächst ist zu berücksichtigen, dass die Gebührenentscheidung auch die Intensität des Wettbewerbs zwischen privaten und öffentlichen Programmanbietern determiniert. 2004 befanden sich die Privatanbieter auf Grund einer seit drei Jahren anhaltenden Werberezession in der Defensive gegenüber den öffentlich-rechtlichen Anstalten (Funk-Korrespondenz, 28. Mai 2004). Diese Entwicklung inspirierte grundsätzliche Zweifel an der substanziellen Rationalität des Regulierungsregimes, weil die öffentlich-rechtlichen Anbieter auf eine Gebührenerhöhung vertrauen konnten, während die privaten Anbieter zu empfindlichen Einschnitten in ihre Programmetats gezwungen waren. Das Regulierungsregime schien ein „crowding out"-Szenario zu begünstigen, in dem die öffentlichrechtlichen Anbieter die Preise auf den Programmbeschaffungsmärkten künstlich hoch halten konnten, um die privaten Anbieter zu marginalisieren. Dieses Szenario beunruhigte vor allem Bayern und Nordrhein-Westfalen als Standortländer privater Medienkonzerne. Die ARD gab diesen Befürchtungen Nahrung, als sie bei der Bewerbung um die Übertragungsrechte der Fußball-Bundesliga Sat.1 überbot (epdmedien, 25.Juni2003).

Darüber hinaus hatte die technologische Dynamik des Mediensektors zur Folge, dass sich die ordnungspolitische Grundsatzfrage nach der Definition des öffentlich-rechtlichen Programmauftrages mit neuer Brisanz stellte, weil die Anstalten ihre Strategie der Angebotsdiversifikation sowohl im digitalen Fernsehen als auch im Internet fortsetzten. So beanspruchten die Anstalten die Existenz eines eigenständigen Programmauftrags im Internet. Tatsächlich wurden sie von den Ländern allerdings in wenig präziser Form ermächtigt, Internet-Angebote zu veranstalten ( $\$ 4$ Abs. 3 ARD-/ZDF-/DLR-StV). Während Vermachtungs- und Missbrauchspotenziale sowie Fragen der Gewährleistung eines chancengleichen Zugangs auch im Bereich der neuen Kommunikations- und Informationstechnologien als gute Gründe für einen gemeinwirtschaftlichen Leistungsauftrag angeführt werden können (Hoffmann-Riem, 2000), war für die Konflikteskalation entscheidend, dass die Anstalten die Konturen ibres Angebotes zunächst im Unklaren ließen und damit politische Gegenaktivitäten auslösten. Ihre unklaren Aussagen weckten nicht nur bei den privaten Konkurrenten Befürchtungen über den Umfang der öffentlich-rechtlichen Internetaktivitäten (VPRT, 2002), die Anstalten traten auch in Konkurrenz mit anderen kommerziellen Inhalteanbietern, etwa Portalbetreibern oder Zeitungsverlagen (Degenhart, 2001). Schließlich provozierten die Versuche der Anstalten, das Internet auch als eine neue Finanzierungsquelle zu nutzen, heftige öffentliche Kritik (Steemers, 2001). 
Die ungelösten ordnungspolitischen Grundsatzfragen verstärkten die Spannungen zwischen dem deutschen Regulierungsregime und dem Regulierungsansatz der Wettbewerbsdirektion der Europäischen Kommission (DG Comp). Diese verfolgt ein ordnungspolitisches Paradigma, das einer Deregulierung der europäischen Fernsehmärkte $\mathrm{zu}$ Lasten der öffentlichen Rundfunkanbieter zugeneigt ist. Für die öffentlichen Rundfunkanbieter war dies besonders gefährlich, weil die DG Comp mit ihren wettbewerbsrechtlichen Kompetenzen das wichtigste Politikinstrument der Europäischen Kommission kontrolliert, mit dessen Hilfe eine Europäisierung von Politikfeldern ggf. auch gegen den Widerstand der EU-Mitgliedstaaten vorangetrieben werden kann (Scharpf, 1999; Harcourt, 2005; Humphreys, 2005). Im Bereich des öffentlichen Rundfunks fokussierten sich die Auseinandersetzungen auf die Frage, ob die staatliche Rundfunkfinanzierung oder Rundfunkgebühren als wettbewerbsverzerrende „Beihilfen“ aufzufassen seien, d. h. letztlich, ob die öffentlichen Rundfunkanbieter staatliche Mittel einsetzten, um kommerzielle Aktivitäten „querzusubventionieren“ und private Anbieter zu marginalisieren (Beck et al., 2004). Da sowohl die EU-Kommission als auch der Europäische Gerichtshof $(\mathrm{EuGH})$ den Beihilfebegriff in anderen Sektoren bereits äußerst extensiv ausgelegt hatten, inspirierten sie wiederholt private Medienunternehmen zur Anstrengung beihilferechtlicher Verfahren gegen öffentliche Rundfunkanbieter (Syvertsen, 2003; Wheeler, 2004). Um zu verhindern, dass die Kommission die Definition des Auftrages der öffentlichen Rundfunkanbieter zu Lasten nationaler Regulierungstraditionen an sich zog, verabschiedeten die Mitgliedstaaten einstimmig einen Protokollanhang zum Vertrag von Amsterdam im Jahr 1997, der festhielt, dass Auftragsdefinition und Finanzierung öffentlicher Rundfunkanbieter in der Kompetenz der Mitgliedstaaten verbleiben sollten (EG-Amtsblatt, C 340, 10. November 1997). Tatsächlich erlegte sich die EU-Kommission in den anhängigen Verfahren Zurückhaltung auf (Harrison \& Woods, 2001; Ward, 2003; Beck et al., 2004). Sie hielt aber an ihren Transparenzbemühungen fest und versuchte, 1999 den Geltungsbereich der Transparenzrichtlinie, die das europäische Beihilferecht für öffentliche Unternehmen konkretisiert, auf öffentliche Rundfunkunternehmen auszudehnen. Nach einem Entwurf der Kommission sollten auch öffentliche Rundfunkunternehmen zu einer nach Geschäftsbereichen getrennten (analytischen) Buchführung für ihre gemeinwirtschaftlichen und kommerziellen Aktivitäten verpflichtet werden, aus der klar hervorgehen sollte, dass staatliche Zuwendungen ausschließlich für die Wahrnehmung öffentlicher Aufgaben verwendet werden (EG-Amtsblatt, C 377, 29. Dezember 1999). In den Erwägungsgründen zur endgültigen Fassung der Transparenzrichtlinie (2000/52/EG) wird freilich auf die Relevanz des Amsterdamer Protokolls für die Anwendung der Transparenzrichtlinie hingewiesen (EG-Amtsblatt, L 193, 29. Juli 2000). In Reaktion auf das Amsterdamer Protokoll nimmt die Kommission keine beihilferechtliche Prüfung der Auftragsdefinition der öffentlichen Rundfunkanbieter selbst vor, sondern beschränkt sich auf die Überprüfung „offensichtlicher Fehler" (EG-Amtsblatt, C 320, 15. November 2001). Obwohl die Kommission damit vom Vorhaben einer umfassenden Auftragsüberprüfung abrückte, weigerten sich die deutschen Bundesländer, die Transparenzrichtlinie für den öffentlichrechtlichen Rundfunk zu implementieren. Sie erklärten, dass das Amsterdamer Protokoll die Finanzierung des öffentlich-rechtlichen Rundfunks grundsätzlich von den Vorschriften des EG-Vertrages befreie (Bundesrats-Drucksache, 663/01).

$\mathrm{Da}$ sich das rundfunkpolitische Verhandlungssystem der Länder nur bedingt responsiv gegenüber den politischen Forderungen der Privatanbieter zeigte, versuchte der Verband Privater Rundfunk und Telekommunikation (VPRT) den Konflikt zwischen EU-Kommission und Ländern zu nutzen und leitete bereits im Vorfeld der Gebühren- 
runde ein Beihilfeverfahren gegen die Bundesrepublik wegen der fehlenden Umsetzung der Transparenzrichtlinie ein (VPRT, 2003; Pelny, 2003). Der VPRT betrachtete seine Beschwerde insoweit als Erfolg verheißend, als er davon ausging, dass das KEF-Verfahren die vom EuGH in seiner Altmark Trans-Entscheidung (EU-Amtsblatt, C 226, 20. September 2003) genauer spezifizierten Anforderungen an Kompensationszahlungen im Hinblick auf Transparenz und Objektivität der Festlegung der Ausgleichszahlungen nicht erfüllen kann (dazu für den VPRT: Pelny, 2005). ${ }^{14}$ In Reaktion auf die europaweiten Beschwerden privater Konkurrenten über eine unkontrollierte Expansion öffentlicher Programmanbieter hatte die EU-Kommission deutlich gemacht, dass sich nach ihrer Auffassung angesichts sinkender Werbeeinnahmen und zunehmender technischer Konvergenz die grundsätzliche Frage nach dem angemessenen Verhältnis zwischen privaten Medien und öffentlich-rechtlichen Rundfunkveranstaltern stellte, weil Letztere in bestimmten Angebotssegmenten ein kostendeckendes privates Angebot unmöglich zu machen schienen (Depypere \& Tigchelaar, 2004; Reding, 2004; Wiedemann, 2004; Pelny, 2005).

(3) Geringe Responsivität des Regulierungsregimes gegenüber neuen Regulierungsproblemen: Von entscheidender Bedeutung war weiterhin, dass die geringe Responsivität des deutschen Regulierungsregimes gegenüber der politischen Kritik an der öffentlich-rechtlichen Expansionspolitik offen zu Tage trat. Die Anstalten ignorierten die deutliche Aufforderung der KEF, ihre Internet-Projekte genauer zu definieren und zu begrenzen (KEF, 2001, Tz. 206-7). Im Gegenteil, ARD und ZDF weiteten ihre OnlineProjekte in ihren Anmeldungen zum 14. Bericht sogar aus. Dies provozierte die deutliche Kritik der KEF (KEF, 2004, Tz. 229), die auch die beträchtliche Angebotsexpansion der letzten zehn Jahre äußerst kritisch kommentierte und offen die Forderung nach einem „Einfrieren“ bzw. einer „Deckelung“ des öffentlich-rechtlichen Programmangebots erhob (KEF, 2004, Tz. 422). Während die Gebührenkommission in ihrem Bericht vom Januar 2004 die den Anstalten zugestandene Gebührenerhöhung drastisch senkte - von angemeldeten 2,01 Euro auf 1,09 Euro -, machte sie deutlich, dass sie von der Existenz weiterer Wirtschaftlichkeitspotenziale in den Anstalten ausging, diese aber auf Grund ihrer eingeschränkten Kompetenzen nicht erschließen konnte (KEF, 2004, Tz. 429).

Zusammenfassend kann festgehalten werden, dass zu Beginn der Gebührenrunde 2004 die Bedingungen für eine Eskalation der Krise des Regulierungsregimes erfüllt waren. Die Gebührenrunde verschaffte Regulierungsdefiziten die notwendige öffentliche Aufmerksamkeit, die aus den kommerziellen Aktivitäten der Rundfunkanstalten resul-

14 An dieser Stelle kann nicht detailliert auf die Implikationen der Altmark Trans-Entscheidung für den öffentlich-rechtlichen Rundfunk eingegangen werden. Während auf der einen Seite in Rechnung zu stellen ist, dass sich der EuGH gegen eine beihilferechtliche Kontrolle sämtlicher Ausgleichszahlungen für gemeinwirtschaftliche Leistungen aussprach, sind die Anforderungen an Kompensationszahlungen außerordentlich hoch. Ungeachtet dessen gehen einige Beobachter davon aus, dass das KEF-Verfahren eine objektive und transparente Berechnung der Ausgleichszahlungen garantiere und Überkompensationen verhindere (König \& Haratsch, 2003). Es ist freilich festzuhalten, dass die KEF sich nicht Fragen der Auftragsdefinition widmet, keine ergebnis- oder performanzorientierte Überprüfung von Ausgleichszahlungen praktiziert, Quersubventionierungen nicht behandelt - erfolgreiche kommerzielle Aktivitäten der Anstalten sogar begrüßt - sowie kaum in der Lage ist, die Kostenmaßstäbe des Altmark Trans-Urteils anzuwenden (s. für den VPRT: Pelny, 2005). Insoweit die genannten Kriterien nicht erfüllt sind, bedeutet dies allerdings nur, dass eine Beihilfe vorliegt, aber nicht, dass diese Beihilfe mit dem freien Markt unvereinbar ist. 
tierenden Interessenkonflikte wurden skandalisiert, während die Entwicklung des Fernsehmarktes und die technologische Dynamik des audiovisuellen Sektors sowohl die Interessenkonflikte zwischen privaten und öffentlich-rechtlichen Anbietern verschärften als auch die grundlegende Frage nach der Performanz des Regulierungsregimes aufwarfen.

\section{Die Eskalation des Legitimitätsverlustes}

Die Kombination dieser Entwicklungen kulminierte 2004 schließlich in einer Krise des Regulierungsregimes, in der es zumindest partiell zu einer Revision etablierter politischer Allianzen kam. Indikatoren dafür sind das gemeinsame Abweichen der Länder von der Gebührenempfehlung der KEF und die Aufgabe der kompletten Verweigerungshaltung der Länder gegenüber der Europäischen Kommission.

Abweichen der Länder von der KEF-Empfehlung: Eine detaillierte Darstellung der auf den 14. KEF-Bericht folgenden langwierigen politischen Auseinandersetzungen um die Gebührenerhöhung kann an dieser Stelle unterbleiben (dazu: Meier, 2005). Eingeleitet wurden die zähen Auseinandersetzungen durch die Drohung eines Gebührenvetos durch die Länder Bayern, Nordrhein-Westfalen und Sachsen im November 2003. Während es sich bei Bayern und Nordrhein-Westfalen um die Länder handelte, die die stärksten Standortinteressen mit dem privaten Rundfunk verbanden, hatte Sachsen bereits im Jahr 2000 einer weiteren Gebührenerhöhung mit Blick auf das geringere Einkommensniveau in Ostdeutschland ablehnend gegenübergestanden. Die drei Länder forderten von den Anstalten Einschnitte im Programmangebot, einen Stellenabbau sowie eine Einschränkung der kommerziellen Aktivitäten (epd medien, 12. November 2003). Die Mehrzahl der SPD-Länder verweigerte sich zwar weit gehenden Einschnitten in das öffentlich-rechtliche Programmangebot (Süddeutsche Zeitung, 14. November 2003), jedoch kritisierten auch Sozialdemokraten offen die Angebotspolitik der Anstalten und erklärten eine Strukturdebatte dann für sinnvoll, wenn dadurch die Belastung für den Gebührenzahler verringert werde (Simonis, 2004). Auf Grund des fehlenden Konsenses über Angebotseinschnitte - einerseits weil eine Beschneidung der Entwicklungschancen der Anstalten auf den Widerstand der Sozialdemokraten traf, andererseits weil die Länder die Rundfunkgebühr weiterhin für die Finanzierung kultur- und industriepolitischer Projekte nutzen wollten -, einigten sich die Länder schließlich nur auf eine marginale Reduktion der Gebührenerhöhung um 23 Cent, was für die Anstalten gegenüber der KEF-Empfehlung eine Einnahmekürzung von jährlich 120 Mio. Euro bedeutete. Für die Entwicklung des Regulierungsregimes ist relevant, dass die Länder sich zwar nicht auf Angebotseinschnitte, aber auf eine Erhöhung des Regulierungsniveaus der Angebotspolitik des öffentlich-rechtlichen Rundfunks verständigten. Die wesentlichen Punkte dieser Einigung, die in $\$ 19$ des neuen Rundfunkstaatsvertrags niedergelegt ist, lauten:

(1) die „Einfrierung“ des Angebotsumfangs auf den Status quo;

(2) die quantitative und inhaltliche Beschränkung der digitalen Programmangebote;

(3) Selbstverpflichtungen sind nun Teil des KEF-Verfahrens, wobei die Länder die Anstalten zu deutlich konkreteren „Selbst“-Verpflichtungen in den Bereichen Online, Marketing und Personal zwangen;

(4) die „bedarfsorientierten“ Bemessungskriterien der KEF wurden dahingehend ergänzt, dass die KEF nun auch die gesamtwirtschaftliche Situation und die Situation der öffentlichen Haushalte berücksichtigen soll.

(5) Schließlich verständigten sich die Länder darauf, bis 2009 eine grundlegende Struk- 
turdebatte zu führen, um eine Gebührenerhöhung entbehrlich zu machen (epd medien, 29. September 2004).

Aufgabe der Verweigerungshaltung gegenüber der Europäischen Kommission: Auch in der Auseinandersetzung mit der EU-Kommission über die Anwendbarkeit des europäischen Beihilferechts auf den deutschen öffentlich-rechtlichen Rundfunk revidierten die Länder partiell ihre bisherige totalablehnende Position, die noch die Bundesratsentschließung zur Anwendbarkeit der Beihilfevorschriften auf den öffentlich-rechtlichen Rundfunk aus dem Jahre 2001 geprägt hat (Bundesrats-Drucksache, 663/01). Dieses Entgegenkommen wurde den Ländern freilich dadurch erleichtert, dass die EU-Kommission eine prinzipielle Auseinandersetzung vermied, indem sie ihren Vorstoß auf ein unkontrolliertes Engagement der Rundfunkanstalten in den neuen Medien beschränkte (DG Comp, 2005). In ihrer vorläufigen Auffassung, die für die endgültige Entscheidung der EU-Kommission jedoch entscheidende Bedeutung haben dürfte, griff die DG Comp allerdings eine ganze Reihe von Kritikpunkten am gegenwärtigen Regulierungsregime des öffentlich-rechtlichen Rundfunks in Deutschland auf, die auch in der aktuellen Gebührendiskussion eine zentrale Rolle spielten bzw. von der KEF im 14. Bericht thematisiert wurden. Darüber hinaus stellte die DG Comp fest, dass der Programmauftrag auch rein kommerzielle Tätigkeiten beinhalte, ein klares Programmkonzept für die neuen Medien fehle, die Mechanismen zur Kontrolle der Auftragserfüllung unzureichend seien und der bestehende Rechtsrahmen wettbewerbswidrige Quersubventionen nicht ausschließe (DG Comp, 2005). Um diese Einwände auszuräumen, schlug die DG Comp eine Reihe von Maßnahmen vor, darunter die weitere Konkretisierung des öffentlichen Auftrages, vor allem im Online- und Digital-Bereich, eine Unterscheidung zwischen gemeinwohlorientierten Aufgaben und kommerziellen Tätigkeiten sowie den Ausschluss wettbewerbswidriger Quersubventionen und eine angemessene ex postKontrolle der Auftragserfüllung (vgl. dazu: DG Comp, 2005, Rz. 244). Mit dem Letzteren ist eine unabhängige nationale Regulierungsbehörde gemeint (IP/05/250).

Die Länder hielten zwar an ihrer prinzipiellen Ablehnung der Anwendbarkeit des Beihilferechts fest, boten aber der EU-Kommission nichtsdestotrotz substanzielle Zugeständnisse an, auch wenn die Länder dabei versuchten, „systemimmanente“ Antworten zu geben. Dies lässt sich auch als implizites Eingeständnis von Regulierungsdefiziten interpretieren. Zu den offerierten Veränderungen am bestehenden Regulierungsregime zählen:

(1) Verbesserungen bei der Auftragsdefinition des öffentlich-rechtlichen Rundfunks, d. h. explizite und detaillierte Vorschriften für kommerzielle und Internet-Aktivitäten sowie stärkere Regulierung der Selbstverpflichtungen der Anstalten;

(2) Stärkere Regulierung des Finanzgebarens und Wettbewerbsverbaltens der öffentlichrechtlichen Anstalten, d. h. Aufnahme gesetzlicher Vorgaben für kommerzielle Tätigkeiten zu Marktkonformität, Fremdvergleichen, analytischer Buchführung sowie effizienterer Kontrolle und größerer Transparenz;

(3) Ausweitung und Stärkung von Kontrollmechanismen, d. h. eine Stärkung der Binnenkontrolle sowie eine begrenzte Ausdehnung der Prüfbefugnisse der Rechnungshöfe und der KEF (Bundesregierung, 2005).

An dieser Stelle kann festgehalten werden, dass die Eskalation der Regulierungskrise zu einer partiellen Revision politischer Standpunkte und zu Bemühungen um eine Reform des Regulierungsregimes geführt haben. Die Implikationen dieser Bemühungen werden im nächsten Punkt diskutiert. 


\section{Diskussion: Grundlegender Umbau des Regulierungsregimes?}

Da die Bedingungen für eine Eskalation der Regulierungskrise erfüllt waren, hätte nach den Überlegungen des „contested governance“-Ansatzes (Ansell \& Vogel, 2006) die Auseinandersetzung um die Regulierung des öffentlich-rechtlichen Rundfunks in eine umfassende Reform des Regulierungsregimes münden müssen. Allerdings erfüllen weder der Gebührenkompromiss der Länder noch die Zugeständnisse der Länder an die EU-Kommission diese Kriterien:

Der wesentliche Effekt der Gebührenintervention der Länder besteht in einer Festschreibung des Status quo-Angebots der Anstalten. Dies stellt immerhin eine partielle Relativierung des bislang dominierenden Regulierungsprinzips einer breit interpretierten Programmautonomie dar. Mit der Aufnahme zusätzlicher Beurteilungsmaßstäbe für die KEF in die Staatsverträge haben die Länder versucht, neue, allerdings wenig konkretisierte Regulierungsprinzipien hinzuzufügen. Darüber hinaus bemühten die Länder sich, die bestehenden Defizite des Regulierungsregimes „systemimmanent“, d. h. verfassungskonform, zu bearbeiten, indem sie die Anstalten noch einmal zu einer Intensivierung ihrer Selbstbindungen aufgefordert haben. Es wird abzuwarten sein, auf welche Weise Anstalten und Länder das Instrumentarium der Selbstbindung mit Leben füllen. An der Fähigkeit der Länder zu der für 2009 vorgesehenen grundlegenden Strukturdebatte kann dagegen begründet gezweifelt werden. Die Gebührenpräferenzen der Länder bleiben widersprüchlich, so dass Einschnitte in das Programmangebot nicht konsensfähig wären.

Die Reaktion der Länder auf das Beihilfeverfahren der EU-Kommission ist ebenfalls vom Versuch einer inkrementellen Reform des Regulierungsregimes geprägt. Während die Anstalten eine stärkere Regulierung ihrer kommerziellen Aktivitäten hinnehmen werden müssen, soll eine Auftragspräzisierung wiederum über Selbstverpflichtungen erreicht werden.

Im Ergebnis ist daher zu konstatieren, dass ein institutioneller "Schneeballeffekt" ausgeblieben ist und das grundlegende Steuerungsproblem einer prekären Anreizstruktur der öffentlich-rechtlichen Rundfunkanstalten weiter besteht. Die inkrementellen Justierungen führen nicht zur Schaffung eines konsistenten Regulierungsregimes für den öffentlich-rechtlichen Rundfunk, sondern fügen dem bestehenden Regulierungsregime additiv eine Reihe neuer Regulierungsprinzipien hinzu. Dies wirft die Frage auf, warum entgegen dem „contested governance“-Modell eine grundlegende Reform des Regulierungsregimes ausgeblieben ist.

Die vorliegende Rekonstruktion lässt zwei Erklärungen für den bevorzugten Rückgriff der Länder auf Selbstverpflichtungen als plausibel erscheinen: Einerseits lässt sich der Rückgriff auf Selbstverpflichtungen als Indikator dafür interpretieren, dass die Länder auf Grund der verfassungsrechtlichen Risiken vor einer grundlegenden Reform des Regulierungsregimes zurückgeschreckt haben. Andererseits scheiterte eine grundlegende Reform aber auch an den mebrdimensionalen Gebührenpräferenzen der Länder. Die legislativen Bemühungen folgen also eher einem Muster der inkrementellen NachJustierungen des Regulierungsregimes. Allerdings liegt die Zukunft des Regulierungsregimes nun nicht mehr allein in der Hand der Länder, da das BVerfG Gelegenheit haben wird, über das letzte Gebührenverfahren zu urteilen. Nach langwierigen internen Debatten entschied sich die ARD im Oktober 2005, gegen den Gebührenkompromiss der Länder vor dem Verfassungsgericht zu klagen, um die politische Unabhängigkeit der Rundfunkanstalten zu verteidigen. Die ARD erklärte diesen Schritt damit, dass die Länder nicht bereit gewesen wären, den von ihr präsentierten Vorschlag für ein neues, „ent- 
politisiertes" Gebührenverfahren zu akzeptieren (Süddeutsche Zeitung, 26. Oktober 2005). Diese Reformüberlegungen belegen, dass die Anstalten zu der Auffassung gelangt sind, dass sich eine zyklisch wiederholte Skandalisierung der Gebührenentscheidung langfristig legitimitätszehrend auswirkt. Der Reformvorschlag der ARD läuft darauf hinaus, die Entpolitisierung der Gebübrenentscheidung gegen deutlich geringere Gebübrenerböbungen zu „tauschen“. Nach den Vorstellungen der ARD soll die Gebührenentwicklung an den Verbraucherpreisindex gekoppelt und im Zwei-Jahres-Rhythmus aktualisiert werden. Die Ratifikation der Gebührenentscheidung durch die Landtage soll „entpolitisiert“ werden, d. h. zu Gunsten einer Verordnung durch die Ministerpräsidenten entfallen ( $A R D$-Presseinformation, 13. Juli 2005). Freilich wäre dieses Verfahren nur insoweit „entpolitisiert“, als parlamentarische Auseinandersetzungen vermieden werden könnten, die in besonderem Maße öffentliche Aufmerksamkeit auf sich ziehen und damit das Ratifikationsverfahren für populistische Kalküle anfällig machen. Allerdings erscheint es keinesfalls gesichert, dass eine Verordnungsgebung durch die Ministerpräsidenten im Hinblick auf solche Entscheidungsparameter weniger „politisiert" wäre.

Die in Abbildung 2 präsentierte „Simulation“ des ARD-Fortschreibungsmodells für die zurückliegenden drei Gebührenperioden macht die weit reichenden Konsequenzen dieser Indexierungsmethode für die Anstalten deutlich. Im Vergleichszeitraum stieg die Gebühr mehr als doppelt so stark an wie die Verbraucherpreise. Nach diesem Modell hätte die Rundfunkgebühr im Jahre 2004 nicht 16,15 Euro, sondern nur 12,59 Euro betragen. Dieses neue Gebührenmodell würde implizieren, dass die Anstalten (a) in erheblichem Masse zu Effizienzsteigerungen gezwungen und (b) in weitaus geringerem Maße zur Erschließung neuer Aufgabenfelder in der Lage sein würden. Vorausgesetzt, dieser Vorschlag wird implementiert, bedeutet dies nichts weniger als einen faktischen Verzicht auf die breit interpretierte Programmantonomie als bislang dominierendem Regulierungsprinzip. In Reaktion auf die Verfassungsklage der ARD, suspendierten die Gebührenkritiker unter den Ländern allerdings die Beratungen über dieses neue Gebührenmodell (Focus, 7. November 2005).

\section{Fazit: Dynamik und Persistenz}

Der vorliegende Beitrag hat sich um den Nachweis bemüht, dass sich die gegenwärtige Krise der Regulierung des öffentlich-rechtlichen Rundfunks als Ergebnis einer Kumulation ungelöster Regulierungsfragen und einer wachsenden Kluft zwischen politischen Leistungserwartungen und der Performanz des existierenden Regulierungsregimes rekonstruieren lässt.

Während sich die Auswirkungen institutioneller Entscheidungen generell schwer abschätzen lassen, weil institutionelle Arrangements stets mit dynamischen gesellschaftlichen Rahmenbedingungen interagieren, wurde hier argumentiert, dass Lücken zwischen politischen Funktionserwartungen und bestehenden Regulierungsregimen insbesondere dann wahrscheinlich werden, wenn der ,institutionelle Architekt“ bestimmten strukturellen Beschränkungen unterliegt und die Kosten für nachträgliche Revisionen seiner Weichenstellungen sehr hoch sind. Es wurde argumentiert, dass diese Voraussetzungen im Fall des öffentlich-rechtlichen Rundfunks vorlagen, weil das BVerfG mit der Stärkung der Programmautonomie eine außerordentlich weit reichende institutionelle Entscheidung getroffen hat, ohne die prekären langfristigen Effekte zu antizipieren oder ein konsistentes Regulierungsregime zu entwerfen. Die Frage, wie politische bzw. gesetzliche Fremd- und autonome Selbstregulierung der Rundfunkanstalten miteinander zu ge- 


\section{Abbildung 2: „Simulation“ des ARD-Modells für frühere Gebübrenperioden}

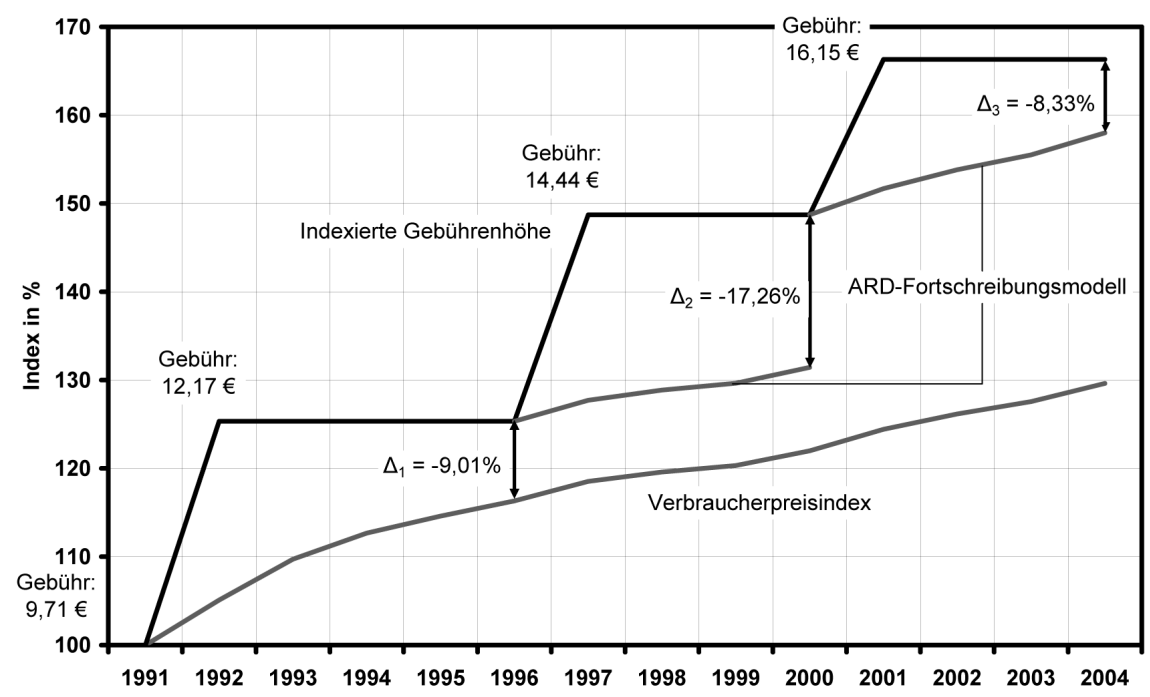

Quelle: Eigene Darstellung auf Basis von Daten des Statistischen Bundesamtes; www.destatis.de/indicators/d/vpi101ad.htm

wichten sind, ist zu einer der schwierigsten Fragen der deutschen Rundfunkordnung avanciert (Ladeur, 2000). Mit Hilfe des analytischen Instrumentariums des PrincipalAgent-Ansatzes konnte gezeigt werden, dass die einseitige Stärkung der Programmautonomie eine Verselbständigung der Anstalten begünstigt, weil die Probleme eines komplexen Zielsystems und einer widersprüchlichen Anreizstruktur ungelöst blieben. Die hohen Veränderungskosten für eine Anpassung des Regulierungsregimes führten dazu, dass sich erhebliche Lücken zwischen politischen Funktionserwartungen und der Performanz des Regulierungsregimes, das den veränderten Regulierungsherausforderungen nicht gewachsen war, auftaten.

Die jüngste Gebührenrunde ließ den politischen Legitimitätsverlust des Regulierungsregimes eskalieren, weil eine Reihe von Eskalationsvoraussetzungen erfüllt war: Entscheidend war die zyklische Aufmerksamkeit für und die Skandalisierung von Regulierungsdefiziten, die mit längerfristigen Interessen- und institutionellen Konflikten korrespondierten. Zentraler Indikator für den Befund des Legitimitätsverlustes ist dabei, dass die Kumulation von Regulierungsdefiziten zu einer Revision politischer Loyalitäten führte. So waren traditionelle Unterstützer des öffentlich-rechtlichen Rundfunks bereit, zu Gunsten einer partiellen Reform des Regulierungsregimes erhebliche verfassungsrechtliche Risiken auf sich zu nehmen, indem sie von der KEF-Empfehlung für eine Gebührenerhöhung abwichen und damit die Voraussetzung für ein neues Verfassungsgerichtsverfahren schufen. Ebenso haben sich die Länder - trotz ihrer weiteren prinzipiellen Ablehnung der Übertragung des europäischen Beihilferegimes auf den öffentlich-rechtlichen Rundfunk - bereit gefunden, der DG Comp in einigen Kritikpunkten am deutschen Regulierungsregime entgegenzukommen. Das Angebot der 
ARD über eine weit reichende Reform des Regulierungsregimes zeigt, dass selbst die Rundfunkanstalten die politischen Akzeptanzgrenzen ihres Wachstums wahrnehmen. Es wird nun abzuwarten sein, ob das BVerfG sich darauf beschränkt, über den Ablauf des letzten Gebührenverfahrens zu entscheiden, oder die Auseinandersetzung zum Anlass nimmt, den Verfahrensgegenstand auszuweiten und grundsätzliche Ausführungen zum Regulierungsregime zu machen (dazu generell etwa: Heun, 1988). Letzteres wird offenbar von den Betroffenen erwartet, die auf prinzipielle Ausführungen zur Zukunft des öffentlich-rechtlichen Rundfunks im digitalen Zeitalter hoffen (vgl. DLR, 2006; ZDF, 2006; VPRT, 2005). Für diesen Fall wird abzuwarten sein, ob das Gericht, das sich unbestrittenermaßen einer besonderen Stellung zwischen Politik und Recht erfreut (Piazolo, 1995), in Reaktion auf die medienpolitische Kritik an der Angebotspolitik der Anstalten und auf die geänderten gesellschaftlichen Rahmenbedingungen zu einer partiellen Revision oder zumindest Präzisierung der Doktrin der Programmautonomie bereit ist. ${ }^{15}$

Für konzeptionelle Überlegungen zur Persistenz von Regulierungsregimen dürfte relevant sein, dass die Auseinandersetzung um den öffentlich-rechtlichen Rundfunk gezeigt haben, dass (a) ein Regulierungsregime eine erhebliche Persistenz aufweisen kann, selbst wenn Funktionsdefizite, wie im Fall des öffentlich-rechtlichen Rundfunks, über einen längeren Zeitraum thematisiert werden, dass aber (b) bei einer entsprechenden $\mathrm{Ku}$ mulation von Regulierungsdefiziten selbst hohe Hürden für institutionelle Anpassungen überwunden werden können. Das bedeutet, dass nicht einmal eine verfassungsrechtliche Legitimation ein defizitäres Regulierungsregime dauerhaft vor institutionellen Krisen und Anpassungen schützen kann. Schließlich kann (c) festgehalten werden, dass die Eskalation einer Regulierungskrise zwar zur Revision politischer Loyalitäten führen kann, aber nicht davon ausgegangen werden kann, dass es sich dabei um eine Totalrevision politischer Präferenzen handelt und notwendig ein "Schneeballeffekt" eintritt. Im untersuchten Fall erwiesen sich die Veränderungskosten des bestehenden Regulierungsregimes weiterhin als sehr hoch, andererseits waren die Länder in der Frage spürbarer Einschnitte in das Angebot des öffentlich-rechtlichen Rundfunks nicht konsensfähig, so dass eine umfassende Reform des Regulierungsregimes ausblieb.

So stellt sich abschließend die Frage, ob die Eskalation von Legitimitätskrisen dazu führt, dass effizientere institutionelle Lösungen gefunden werden (auch wenn diese Frage angesichts der ausstehenden Verfassungsgerichtsentscheidung nur vorläufig beantwortet werden kann). Die vorliegende Fallstudie nährt eher Skepsis im Hinblick auf die politische Selektion effizienter institutioneller Lösungen. Durch die jüngsten Justierungen ist das bestehende System fragmentierter Einflussbeziehungen und konkurrierender Regulierungsziele weiter verkompliziert worden, selbst wenn die Auftragsdefinitionen der Anstalten präzisiert und die Transparenz ihres Finanzgebarens erhöht werden sollten. Die vorgesehene Einfrierung des Programmangebotes und die (dynamische) Deckelung der Gebührenhöhe werden zwar den Bewegungsspielraum der Anstalten empfindlich einschränken, die grundlegenden ordnungspolitischen Fragen bleiben jedoch ungeklärt, ebenso werden Zielkonflikte, prekäre Anreizstrukturen und das Problem der eingeschränkten Strategiefähigkeit und Regulierungskapazität der binnenpluralistischen Aufsichtsgremien vorerst bestehen bleiben. Ungeachtet des ausstehenden Verfassungs-

15 Damit soll nicht suggeriert werden, das Gericht handele wie ein politischer Akteur. Die pluralistische Theorie der Verfassungsrechtsprechung geht jedoch davon aus, dass das Verfassungsgericht durchaus „adressatenspezifische“ Erwägungen bei seinen Entscheidungen berücksichtigt. Allerdings ist diese These empirisch kontrovers (vgl. nur: Ebsen, 1985). 
gerichtsurteils kann daher konstatiert werden, dass das Regulierungsregime für den öffentlich-rechtlichen Rundfunk weiterhin genügend Spannungen beinhaltet, die eine künftige Institutionendynamik auslösen könnten.

\section{Literatur}

Alchian, A. A.; Demsetz, H. (1973): The property rights paradigm. Journal of Economic History 33, S. 16-27.

Anschlag, D. (2004): Für ein paar Cent weniger: Das Gefeilsche um die Rundfunkgebühren. FunkKorrespondenz (40), S. 3-4.

Ansell, C.; Vogel, D. (2006): The contested governance of European food safety. In: Ansell, C.; Vogel, D., (Eds.): What's the beef: The contested governance of European food safety regulation, Boston: MIT Press (im Druck).

Bartels, R. (1997): Fernsehnachrichten im Wettbewerb, Köln et al.: Böhlau.

Beck, D.; Münger, T.; Pitum, S.; Sauer, J. (2004): Service Public unter Druck? Die Auswirkungen der EU-Transparenzrichtlinie auf den öffentlich-rechtlichen Rundfunk, Zürich: Rüegger.

Bethge, H. (1996): Die verfassungsrechtliche Position des öffentlich-rechtlichen Rundfunks in der dualen Rundfunkordnung, Baden-Baden: Nomos.

Blumler, J. G. (1993): Meshing money with mission. European Journal of Communication 8, S. 403424.

Brandt, W. (1989): Strategien für Rundfunkanstalten, Frankfurt am Main et al.: Lang.

Bruns, T.; Marcinkowski, F. (1996): Konvergenz Revisited. Neue Befunde zu einer älteren Diskussion. Rundfunk und Fernsehen 44, S. 461-478.

Bullinger, M. (1999): Die Aufgaben des öffentlichen Rundfunks: Wege zu einem Funktionsanftrag. Gütersloh: Bertelsmann-Stiftung.

Bundesregierung (2005): Mitteilung der Bundesregierung an die Europäische Kommission: Staatliche Beibilfen E 3/2005 (ex CP 232/2002, CP 2/2003, CP 195/2004 und CP 234/2004): Die Finanzierung der öffentlich-rechtlichen Rundfunkanstalten - Deutschland, Berlin: Bundesregierung.

Degenhart, C. (2001): Der Funktionsauftrag des öffentlich-rechtlichen Rundfunks in der „Digitalen Welt“, Leipzig: Universität Leipzig.

Deiss, R. (2002): The European TV broadcasting market. Statistics in Focus, Theme 4, 24/2002, Brüssel; Eurostat.

Depypere, S.; Tigchelaar, N. (2004): The commission's state aid policy on activities of public service broadcasters in neighbouring markets. Competition Policy Newsletter (2), S. 19-22.

DG Comp (2005): Vorläufige Auffassung der Generaldirektion Wettbewerb hinsichtlich der Vereinbarkeit des bestehenden Systems über die Finanzierung des öffentlichen Rundfunks in Deutschland mit dem Gemeinsamen Markt. epd medien (21), S. 7-32.

DLR (2006): Finanzierung der Grundversorgung mit Information und Kultur sicherstellen: Deutschlandradio-Stellungnabme zur Verfassungsbeschwerde des öffentlich-rechtlichen Rundfunks. Berlin und Köln: DLR www.dradio.de/wir/presse/478547/ (12.04.2006).

Dussel, K. (1995): Die Interessen der Allgemeinheit vertreten: Die Tätigkeit der Rundfunk-und Verwaltungsräte von Südwestfunk und Süddeutschen Rundfunk, Baden-Baden: Nomos.

Eberle, C.-E. (2004): Der Missbrauch des Wettbewerbsrechts zeugt von Zynismus. promedia (9), S. $5-6$.

Eberlein, B.; Grande, E. (2005): Beyond delegation: Transnational regulatory regimes and the EU regulatory state. Journal of European Public Policy 12, S. 89-112.

Ebsen, I. (1985): Das Bundesverfassungsgericht als Element gesellschaftlicher Selbstregulierung: eine pluralistische Theorie der Verfassungsgerichtsbarkeit im demokratischen Verfassungsstaat, Berlin: Duncker \& Humblot.

Eisenhardt, K. M. (1989): Agency theory: An assessment and review. Academy of Management Review 14 , S. $57-74$.

Europäische Kommission (2003): Cinema, TV and radio in the EU: Statistics on audiovisual services: Data 1980-2002, Brüssel: Europäische Kommission. 
Fama, E.; Jensen, M. C. (1983): Separation of ownership and control. Journal of Law E Economics 26, S. 310-325.

Fritz, R. (1977): Massenmedium Rundfunk: Die rechtliche Stellung der Rundfunkräte und ibre tatsächliche Einflussnahme auf die Programmgestaltung, Frankfurt am Main: Univ.-Diss.

Gellner, W. (1990): Ordnungspolitik im Fernsebwesen, Frankfurt am Main: Lang.

Gilardi, F.; Braun, D. (2002): Delegation aus der Sicht der Prinzipal-Agent-Theorie. Politische Vierteljabresschrift 43, S. 147-161.

Harcourt, A. (2005): The European Union \& the regulation of media markets, Manchester, New York: Manchester University Press.

Hargreaves Heap, S. P. (2005): Television in a digital age: What role for public service broadcasting? Economic Policy 41, S. 111-158.

Harrison, J.; Woods, L. M. (2001): Defining European public service broadcasters. European Journal of Communication 16, S. 477-504.

Hart, O. D.; Holmstrom, B. R. (1987): The theory of contracts. In: Bewley, T. (ed.): Advances in economic theory, Cambridge: Cambridge University Press, S. 71-155.

Hennig, J. (1981): Rundfunkräte auf dem Prüfstand. Bonn: Bundeszentrale für politische Bildung. Hesse, A. (1999): Rundfunkrecht, 2. Aufl., München: Beck.

Heun, W. (1988): Der Zugang zum BVerfG: Zugangsfilter, Steuerungsmöglichkeiten des Gerichts, Mobilisierung. In: Ellermann, R.; Gawron, T. und Rogowski, R. (Hrsg.): Verfassungsgerichte im Vergleich, Gummersbach: Friedrich-Naumann-Stiftung, S. 203-236.

Hoffmann, R. (1975): Rundfunkorganisation und Rundfunkfreibeit: Die Entwicklung von Organisations- und Machtstrukturen im Westdeutschen Rundfunk Köln und das Selbstverständnis der Programmacher. Köln: Spiess.

Hoffmann-Riem, W. (1994): Stadien des Rundfunk-Richterrechts. In: Jarren, O. (Hrsg.): Medienwandel-Gesellschaftswandel? Zehn Jahre dualer Rundfunk in Deutschland, Berlin: Vistas, S. 17-34.

Hoffmann-Riem, W. (2000): Regulierung der dualen Rundfunkordnung: Grundfragen. Baden-Baden: Nomos.

Holmstrom, B. R.; Milgrom, P. (1991): Multitask principal-agent analyses. Journal of Law, Economics $\mathcal{E}$ Organization 7 (Special Issue), S. 24-52.

Holznagel, B. (1999): Der spezifische Funktionsauftrag des Zweiten Deutschen Fernsehens (ZDF), Mainz: ZDF.

Holznagel, B.; Vesting, T. (1999): Spartenprogramme und Zielgruppenprogramme im öffentlichrechtlichen Rundfunk, insbesondere im Hörfunk, Baden-Baden: Nomos.

Humphreys, P. (2005): The EU and audiovisual regulation: An agency for de-regulation or re-regulation: Paper presented at the 3rd ECPR in Budapest, 8-10 September 2005.

Jarass, H. D. (1997): Online-Dienste und Funktionsbereich des Zweiten Deutschen Fernsehens, Mainz: ZDF.

Jarren, O. (2001): „Mediengesellschaft“ - Risiken für die politische Kommunikation. Aus Politik und Zeitgeschichte (B 41-42), S. 10-19.

Jensen, M. C. (1983): Organization theory and methodology. The Accouting Review 58, S. 319 339.

Kammann, U. (2003): Wellenbrecher: Ersticktes Programm. epd medien (95), S. 3-5.

Kayser, H. J. (1993): Controlling für Rundfunkanstalten, Baden-Baden: Nomos.

$\mathrm{KEF}$ (2001) 13. KEF-Bericht, Mainz: KEF.

$\mathrm{KEF}$ (2004): 14. KEF-Bericht, Mainz: KEF.

Kepplinger, H. M.; Hartmann, T. (1989): Stachel oder Feigenblatt? Rundfunk-und Fernsebräte in der Bundesrepublik Deutschland, Frankfurt am Main: IMK.

Kiewiet, D. R.; McCubbins, M. C. (1991): The logic of delegation. Chicago: University of Chicago Press.

Kingdon, J. W. (1995): Agendas, Alternatives and Public Policies, 2nd edn. New York: HarperCollins.

König, C.; Haratsch, A. (2003): Die Rundfunkgebühren auf dem Prüfstand des Altmark Trans-Urteils des Europäischen Gerichtshofs. Zeitschrift für Urbeber- und Medienrecht 47, S. 804-812.

König, E. (1983): Zielorientierte externe Rechnungslegung für die öffentlich-rechtlichen Rundfunk- 
anstalten in der Bundesrepublik Deutschland: Überlegungen zu einer an den Informationsinteressen der Rundfunkteilnehmer ausgerichteten externen Rechnungslegung, Köln: Beck.

Krüger, U. M. (1992): Programmprofile im dualen Fernsehsystem 1985-1990: Eine Studie der $A R D / Z D F-M e d i e n k o m m i s s i o n$, Baden-Baden: Nomos.

Krüger, U. M. (1996): Tendenzen in den Programmen der großen Fernsehsender 1985 bis 1995: Elf Jahre Programmanalyse im dualen Rundfunksystem. Media Perspektiven (8), S. 418-440.

Krüger, U. M. (2001): Zur medienpolitischen Instrumentalisierung der Konvergenzthese von Heribert Schatz. In: Abromheit, H.; Nieland, J.-U.; Schierl, T. (Hrsg.): Politik, Medien, Technik, Opladen: Westdeutscher Verlag, S. 187-206.

Ladeur, K.-H. (1978): Rundfunkverfassung und Pluralismus. Rundfunk und Fernseben 26, S. 141163.

Ladeur, K.-H. (2000): Der „Funktionsauftrag“ des öffentlich-rechtlichen Rundfunks - auf „Integration" festgelegt oder selbst definiert? Medien und Kommunikationswissenschaft 48, S. 93106.

Lilienthal, V. (2004a): Ausverkauf: ZDF-Kooperationen mit Dritten. epd medien (19), S. 3-14.

Lilienthal, V. (2004b): Um ein Ritual reicher: Die Selbstverpflichtungen von ARD, ZDF und DLR. epd medien (73), S. 3-4.

Lilienthal, V. (2005): Die Schwarzarbeit des Fernsehens: Der Bavaria-Skandal und die Folgen. epd medien (44), S. 3-5.

Majone, G. (2001): Nonmajoritarian institutions and the limits of democratic governance. Journal of Institutional \& Theoretical Economics 157, 57-78.

March, J. G.; Olsen, J. P (1989): Rediscovering institutions: The organizational basis of politics, New York: Free Press.

Marcinkowski, F.; Bruns, T. (1996): Politische Magazine im dualen Fernsehen: Probleme einer Unterscheidung. In: Schatz, H. (Hrsg.): Fernsehen als Objekt und Moment des sozialen Wandels: Faktoren und Folgen der aktuellen Veränderungen des Fernsehens, Opladen: Westdeutscher Verlag, S. 255-286.

Mattern, K.; Künstner, T. (1998): Fernsehsysteme im internationalen Vergleich. In: Hamm, I., (Hrsg.) Die Zukunft des dualen Systems: Aufgaben des dualen Rundfunkmarktes im internationalen Vergleich, Gütersloh: Bertelsmann Stiftung, S. 15-204.

Mayntz, R. (2002): Zur Theoriefähigkeit makro-sozialer Analysen. In: Mayntz, R. (Hrsg.): Akteure - Mechanismen - Modelle: Zur Theoriefähigkeit makro-sozialer Analysen. Frankfurt am Main, New York: Campus, S. 7-43.

McCubbins, M. C.; Schwartz, T. (1984): Congressional oversight overlooked: Police patrols versus fire alarms. American Journal of Political Science 28, S. 165-179.

Meier, H. E. (2003a): Strategieanpassungsprozesse im öffentlich-rechtlichen Fernsehen, Berlin: Vistas.

Meier, H. E. (2003b): Beyond convergence. European Journal of Communication 18, S. 337-365.

Meier, H. E. (2005): „Für ein paar Cent weniger"?: Ein Beitrag zur aktuellen Rundfunkgebührenpolitik, Köln: Institut für Rundfunkökonomie an der Universität zu Köln (Arbeitspapiere des Instituts für Rundfunkökonomie an der Universität zu Köln; 197).

Miller, G. (2000): Rational choice and dysfunctional institutions. Governance 13, S. 535-547.

Moe, T. M. (1984): The new economics of organization. American Journal of Political Science 28, S. 739-77.

Neun, A. (2002): Öffentlich-rechtlicher Rundfunk: Grenzen des Wachstums, Berlin: Duncker \& Humblot.

North, D. C. (1989): A transaction cost approach to the historical development of polities \& economies. Journal of Institutional and Theoretical Economics 145, S. 661-668.

North, D. C. (1990): Institutions, institutional change, and economic performance, Cambridge, New York: Cambridge University Press.

Ory, S. (1994): Das Dilemma mit den Rundfunkgebühren - Oder: Prozessuraler Grundrechtsschutz. Zeitschrift für Urheber- und Medienrecht 38, S. 610-616.

Ossenbühl, F. (1981): Programmnormen im Rundfunkrecht. Rundfunkrecht, München: Beck, S. 170. 
Pantenburg, U. (1996): Die Organisation der Leitungsspitze von Rundfunkanstalten, Baden-Baden: Nomos.

Pelny, S. (2003): Das Verfahren der EU-Kommission gegen Dänemark. Zeitschrift für Urheberund Medienrecht 47, S. 643-653.

Pelny, S. (2005): Das Verfahren der EU-Kommission gegen Dänemark: Ein Menetekel für die Länderregierungschefs in Deutschland? Zeitschrift für Urheber- und Medienrecht 49, S. 22-29.

Perrow, C. (1986): Complex organizations, 3rd ed., New York: Random House.

Pfetsch, B. (1996): Konvergente Fernsehformate in der Politikberichterstattung? Eine vergleichende Analyse öffentlich-rechtlicher und privater Programme 1985/86 und 1993. Rundfunk und Fernseben 44, S. 479-498.

Piazolo, M. (Hrsg.) (1995): Das Bundesverfassungsgericht: Ein Gericht im Schnittpunkt von Recht und Politik, Mainz: v. Hase und Koehler.

Pierson, P.; Skocpol, T. (2002): Historical institutionalism in contemporary political science. In: Katznelson, I.; Helen, M. (eds.): Political science: The state of the discipline. New York: Norton, S. 693-721.

Pierson, P. (2004): Politics in time: History, institutions, and social analysis, Princeton: Princeton University Press.

Pratt, J. W.; Zeckhauser, R. J. (1985): Principals and agents: An overview. In: Pratt, J. W.; Zeckhauser, R. J. (eds.): Principals and agents: The structure of business, Boston, MS: Harvard Business School Press, S. 1-36.

Prodoehl, H. G. (1989): Modell und Wirklichkeit: Legitimationsprobleme des öffentlich-rechtlichen Rundfunks in der Bundesrepublik Deutschland. Politische Vierteljabresschrift 30, S. 273 291.

Reding, V. (2004): Praktische Konkordanz: Europäische Visionen einer pluralen Medienordnung. epd medien (20), S. 24-31.

Rueschemeyer, D., Stephens, J. D. (1997): Comparing historical sequences. Comparative Social Research 16, S. 55-72.

Scharpf, F. W. (2000): Interaktionsformen: Akteurzentrierter Institutionalismus in der Politikforschung. Opladen: Westdeutscher Verlag.

Scharpf, F. W. (1999): Regieren in Europa: Effektiv und demokratisch? Frankfurt am Main: Campus.

Schatz, H. (1994): Das duale Rundfunksystem in modernisierungstheoretischer Sicht. In: Holgersson, S.; Jarren, O.; Schatz, H. (Eds.): Dualer Rundfunk in Deutschland: Beiträge zu einer Theorie der Rundfunkentwicklung, Münster: Lit, S. 193-204.

Schatz, H.; Immer, N.; Marcinkowski, F. (1989): Der Vielfalt eine Chance? Empirische Befunde zu einem zentralen Argument für die „Dualisierung“ des Rundfunks in der Bundesrepublik Deutschland. Rundfunk und Fernseben 37, S. 5-25.

Schulz, W. (Hrsg.) (2002): Staatsferne der Aufsichtsgremien öffentlich-rechtlicher Rundfunkanstalten. Materialien zur Diskussion um eine Reform. Hamburg: Hans-Bredow-Institut (Arbeitspapiere des Hans-Bredow-Instituts für Medienforschung an der Universität Hamburg; 12).

Simonis, H. (2004): Der Gesamtkörper der Anstalten ist zu groß und zu teuer geworden. promedia (1), S. 4-6.

Spallek, C. (2001): Perspektiven des öffentlich-rechtlichen Rundfunks in der Informationsgesellschaft. Köln: Institut für Rundfunkökonomie an der Universität zu Köln (Arbeitspapiere des Instituts für Rundfunkökonomie an der Universität zu Köln; 142).

Steemers, J. (2001): In search of a third way. Canadian Journal of Communication 26, S. 1-15.

Stock, M. (1997): Medienpolitik auf neuen Wegen - weg vom Grundgesetz? Rundfunk und Fernseben 45, S. 141-172.

Stock, M. (2001): Innere Medienfreiheit: Ein modernes Konzept der Qualitätssicherung, Baden-Baden: Nomos.

Stoiber, E. (2004): Nur ein klares öffentlich-rechtliches Profil verschafft der Pflichtgebühr Akzeptanz. promedia (5), S. 4.

Stolte, D. (1997): Bleibt Fernsehen Fernsehen?, Mainz: ZDF.

Syvertsen, T. (2003): Challenges to public television in the era of convergence \& commercialization. Television E New Media 4, S. 155-175. 
Thelen, K. (1999): Historical institutionalism in comparative politics. Annual Review of Political Science 2, S. 369-404.

Tonnemacher, J. (1996): Kommunikationspolitik in Deutschland: Eine Einführung, Konstanz: UVK.

VPRT (2002): Erster Diskussionsbeitrag des Verbandes Privater Rundfunk und Telekommunikation e. V. (VPRT) für eine Selbstverpflichtung der öffentlich-rechtlichen Rundfunkanstalten, Berlin: VPRT.

VPRT (2003): Beschwerde bei der Europäischen Kommission wegen Online-und E-Commerce-Aktivitäten von ARD und ZDF eingereicht, Berlin: VPRT.

VPRT (2005): Anrufe des Bundesverfassungsgerichts Ohrfeige für die Länder: Private Medienwirtschaft erhofft sich Aussagen des Bundesverfassungsgerichts zur Neudefinition des Auftrags für ARD und ZDF. Berlin: VPRT, www.vprt.de/dateien/pm_20050614_oerr_karlsruhe.pdf (12.04.2006).

Ward, D. (2003): State aid or band aid? An evaluation of the European Commission's approach to public service broadcasting. Media, Culture E Society 25, S. 233-250.

Warner, W. K.; Havens, A. E. (1968): Goal displacement and intangibility of organizational goals. Administrative Science Quarterly 12, S. 539-555.

Wheeler, M. (2004): Supranational regulation: Television and the European Union. European Journal of Communication 19, S. 349-369.

Wiedemann, V. (2004): Geschäftserwartungen der Wettbewerber: Wendungen der EU-Rundfunkpolitik. epd medien (68), S. 3-12.

ZDF (1994): Das ZDF vor den Herausforderungen des digitalen Fernsehens, Mainz: ZDF.

ZDF (2006): ZDF geht nach Karlsrube. Schächter: „Es geht um zentrale Weichenstellungen für das Fernseben in Deutschland", Mainz: ZDF, www.zdf.de/ZDFde/inhalt/20/0,1872,3907476,00. html (12.04.2006). 